\title{
International Society for the Study of Women's Sexual Health Clinical Practice Guideline for the Use of Systemic Testosterone for Hypoactive Sexual Desire Disorder in Women
}

\author{
Sharon J. Parish, MD, ${ }^{1,2}$ James A. Simon, MD, ${ }^{3}$ Susan R. Davis, MBBS, PhD, ${ }^{4}$ Annamaria Giraldi, MD, PhD, 5 \\ Irwin Goldstein, MD, ${ }^{7,8}$ Sue W. Goldstein, BA, CSE, ${ }^{8}$ Noel N. Kim, PhD, ${ }^{9}$ Sheryl A. Kingsberg, PhD, ${ }^{10}$ \\ Abraham Morgentaler, MD, ${ }^{11}$ Rossella E. Nappi, MD, PhD, ${ }^{12}$ Kwangsung Park, MD, PhD, ${ }^{13}$ \\ Cynthia A. Stuenkel, MD, ${ }^{14}$ Abdulmaged M. Traish, $\mathrm{PhD},{ }^{15}$ and Linda Vignozzi, MD ${ }^{16,17}$
}

\begin{abstract}
Background: The Global Consensus Position Statement on the Use of Testosterone Therapy for Women (Global Position Statement) recommended testosterone therapy for postmenopausal women with hypoactive sexual desire disorder (HSDD).

Aim: To provide a clinical practice guideline for the use of testosterone including identification of patients, laboratory testing, dosing, post-treatment monitoring, and follow-up care in women with HSDD.

Methods: The International Society for the Study of Women's Sexual Health appointed a multidisciplinary panel of experts who performed a literature review of original research, meta-analyses, review papers, and consensus guidelines regarding testosterone use in women. Consensus was reached using a modified Delphi method.

Outcomes: A clinically useful guideline following a biopsychosocial assessment and treatment approach for the safe and efficacious use of testosterone in women with HSDD was developed including measurement, indications, formulations, prescribing, dosing, monitoring, and follow-up.

Results: Although the Global Position Statement endorses testosterone therapy for only postmenopausal women, limited data also support the use in late reproductive age premenopausal women, consistent with the International Society for the Study of Women's Sexual Health Process of Care for the Management of HSDD.
\end{abstract}

Departments of ${ }^{1}$ Psychiatry and ${ }^{2}$ Medicine, New York Presbyterian Hospital/Westchester Behavioral Health Center, White Plains, New York, USA.

${ }^{3}$ IntimMedicine Specialists, George Washington University School of Medicine, Washington, District of Columbia, USA.

${ }^{4}$ Women's Health Research Program, Department of Epidemiology and Preventive Medicine, School of Public Health and Preventive Medicine, Monash University, Melbourne, Australia.

${ }^{5}$ Sexological Clinic, Psychiatric Center, Copenhagen, Denmark.

${ }^{6}$ Department of Clinical Medicine, University of Copenhagen, Copenhagen, Denmark.

${ }^{7}$ Sexual Medicine, Alvarado Hospital, San Diego, California, USA.

${ }^{8}$ San Diego Sexual Medicine, San Diego, California, USA.

${ }^{9}$ Institute for Sexual Medicine, San Diego, California, USA.

${ }^{10}$ University Hospitals Cleveland Medical Center, Case Western Reserve University School of Medicine, Cleveland, Ohio, USA.

${ }^{11}$ Men's Health Boston, Beth Israel Deaconess Medical Center, Harvard Medical School, Chestnut Hill, Massachusetts, USA.

${ }^{12}$ Department of Clinical, Surgical, Diagnostic and Pediatric Sciences, Center for Reproductive Medicine, Gynecological Endocrinology and Menopause, Obstetrics and Gynecology Unit, IRCCS S. Matteo Foundation, University of Pavia, Pavia, Italy.

${ }^{13}$ Department of Urology, Chonnam National University Medical School, Gwangju, Korea.

${ }^{14}$ Department of Medicine, UC San Diego School of Medicine, La Jolla, California, USA.

${ }^{15}$ Departments of Biochemistry and Urology, Boston University School of Medicine, Boston, Massachusetts, USA.

${ }^{16}$ Andrology, Women's Endocrinology \& Gender Incongruence Unit, Department of "Excellence" Experimental and Clinical Biomedical Sciences "Mario Serio"-Careggi Hospital-University of Florence, Florence, Italy.

${ }^{17}$ Consorzio Interuniversitario I.N.B.B., Rome, Italy.

(C) 2021 The Author(s). Published by Elsevier Inc. on behalf of International Society for Sexual Medicine; Informa UK Limited, trading as Taylor \& Francis Group, on behalf of International Menopause Society, and Journal of Women's Health; Mary Ann Liebert, Inc., publishers. This is an Open Access article distributed under the terms of the Creative Commons Attribution-NonCommercial-NoDerivatives License (http://creativecommons.org/licenses/by-nc-nd/4.0/), which permits non-commercial re-use, distribution, and reproduction in any medium, provided the original work is properly cited, and is not altered, transformed, or built upon in any way. 
Systemic transdermal testosterone is recommended for women with HSDD not primarily related to modifiable factors or comorbidities such as relationship or mental health problems. Current available research supports a moderate therapeutic benefit. Safety data show no serious adverse events with physiologic testosterone use, but long-term safety has not been established. Before initiation of therapy, clinicians should provide an informed consent. Shared decision-making involves a comprehensive discussion of off-label use, as well as benefits and risks. A total testosterone level should not be used to diagnose HSDD, but as a baseline for monitoring. Government-approved transdermal male formulations can be used cautiously with dosing appropriate for women. Patients should be assessed for signs of androgen excess and total testosterone levels monitored to maintain concentrations in the physiologic premenopausal range. Compounded products cannot be recommended because of the lack of efficacy and safety data.

Clinical Implications: This clinical practice guideline provides standards for safely prescribing testosterone to women with HSDD, including identification of appropriate patients, dosing, and monitoring.

Strengths \& Limitations: This evidence-based guideline builds on a recently published comprehensive metaanalysis and the Global Position Statement endorsed by numerous societies. The limitation is that testosterone therapy is not approved for women by most regulatory agencies, thereby making prescribing and proper dosing challenging. Conclusion: Despite substantial evidence regarding safety, efficacy, and clinical use, access to testosterone therapy for the treatment of HSDD in women remains a significant unmet need.

Keywords: systemic testosterone, hypoacitve sexual desire disorder, clinical practice guideline, female sexual dysfunction, ISSWSH

\section{Introduction}

$\mathbf{T}$ ESTOSTERONE HAS BEEN an important evidence-based, albeit controversial, therapy for women with hypoactive sexual desire disorder (HSDD). Over the past 2 decades, multiple publications consisting of original research, reviews, and meta-analyses have supported the use of testosterone therapy for HSDD in postmenopausal women. ${ }^{1-5}$ The Global Consensus Position Statement on the Use of Testosterone Therapy for Women (Global Position Statement) was recently published simultaneously in 4 journals, with authors representing 10 societies including the International Society for the Study of Women's Sexual Health (ISSWSH) and endorsed by the ISSWSH and 10 other sexual medicine, endocrine, obstetrics and gynecology, and menopause societies. $^{6-9}$ The Global Position Statement is the most comprehensive, evidence-based guideline to date, superseding all previous guidelines. It provided clinical guidance regarding the use of testosterone therapy in women, examining the effect on sexual function; well-being, mood, and cognition; musculoskeletal effects; cardiovascular and breast health; as well as androgenic side effects and adverse events. The conclusions were developed based on the systematic review and meta-analysis of the benefits and risks of testosterone therapy in women, ${ }^{5}$ and the clinical practice recommendations were based on the expert opinion and the consensus of the panelists. According to the Global Position Statement, the sole evidence-based indication for testosterone therapy is HSDD in postmenopausal women, using a biopsychosocial assessment and treatment model. ${ }^{6}$ The Global Position Statement provides the evidence and standard of care for the use of testosterone in women and serves as the basis for this clinical guidance document. There remains a need for a consensus clinical practice guideline that provides a comprehensive management strategy for the use of systemic testosterone in women with HSDD. The purpose of the present article is to provide specific recommendations re- garding identification of patients, laboratory testing, dosing, monitoring, and follow-up care in the consideration of testosterone therapy for HSDD in women.

\section{Background on Regulatory Issues}

Government-approved testosterone products for HSDD in women are not currently available in the United States or approved by any national regulatory authority except in Australia. 2 transdermal testosterone products, a patch and a transdermal gel, underwent Food and Drug Administration (FDA) evaluation more than 10 years ago. The gel (LibiGel, BioSante Pharmaceuticals, Inc., Lincolnshire, IL) failed to show efficacy greater than placebo; a higher than expected placebo response was attributed to regular clinic visits, daily diary reminders, and subjects' expectations of improvement. ${ }^{10}$ However, efficacy over placebo was successfully demonstrated for the patch (Intrinsa, Procter \& Gamble, Cincinnati, $\mathrm{OH})$ for treatment of HSDD. However, the FDA declined approval based on safety concerns raised by its advisory committee, despite the absence of signals supporting those safety concerns in the regulatory submission. ${ }^{10}$ Despite the lack of evidence for cardiovascular events or breast cancer in randomized, placebo-controlled clinical trials of testosterone in postmenopausal women with HSDD, ${ }^{11,12}$ the regulators had concerns about these risks, given the results of the Women's Health Initiative. ${ }^{13,14}$ However, shortly after the FDA's decision, the European Medicines Evaluation Agency (now called the European Medicines Agency) approved the Intrinsa testosterone patch for HSDD but limited the indication to women with surgically induced menopause taking concomitant estrogen therapy. Unfortunately, it was withdrawn from the European market by the manufacturer because of poor sales. Therefore, due to the lack of government-approved testosterone products for women, clinicians generally have resorted to using products off-label that are approved for men and compounded formulations without a clear standard of clinical care. 


\section{Methods}

This guideline was developed with the administrative support of the ISSWSH and the contributions from a multidisciplinary panel of international experts. The panel, consisting of 16 researchers and clinicians, who were the ISSWSH members and nonmembers, convened to review and discuss clinical management strategies for the use of testosterone using a modified Delphi method. ${ }^{15}$ In preparation, the panelists conducted an evidence-based literature review that included original research, meta-analyses, reviews, and clinical practice and consensus guidelines regarding testosterone in women. Where guidance is not provided in the Global Position Statement, previously published guidelines have been used. At the consensus meeting, the panelists presented summaries of the current literature; discussion of testosterone measurement, formulations, and indications for treatment; and developed consensus for this clinical guideline.

\section{Classification of HSDD}

In the Diagnostic and Statistical Manual of Mental Disorders (DSM) IV-TR, HSDD is defined as "Persistent or recurrent deficiency or absence of sexual fantasies and desire for sexual activity with marked distress or interpersonal difficulty not otherwise accounted for by a general medical or psychiatric condition. HSDD may be primary or secondary, lifelong or acquired, and generalized or situational." 16 In the DSM-5, HSDD and female sexual arousal disorder were merged into a single diagnosis, female sexual interest and arousal disorder. ${ }^{17}$ However, female sexual interest and arousal disorder as a diagnostic entity has been controversial and to date has not been used in the pharmacological interventional clinical trials of testosterone in women. ${ }^{18}$ Although HSDD may overlap with other sexual health disorders, it is a distinct diagnostic category. ${ }^{19}$ Based on this evidence, HSDD is the desire dysfunction specified in the ISSWSH nomenclature, the Fourth International Consultation on Sexual Medicine classification, the International Classification of Diseases-10, and the upcoming International Classification of Diseases-11. ${ }^{18-21}$ All the published randomized, controlled clinical trials of testosterone vs placebo used a definition of HSDD consistent with the DSM IV. ${ }^{18,19,21-23}$ In this article, we explore in depth the indications and use of testosterone treatment for generalized, acquired HSDD.

\section{Epidemiology of Menopause and Impact on Sexual Health}

The number of perimenopausal and postmenopausal women is increasing, and HSDD is a significant problem in general and of particular concern in this population. ${ }^{24,25}$ In a random population study of 3,350 women aged 40-80 years in China, Taiwan, South Korea, Japan, Thailand, Singapore, Malaysia, Indonesia, and the Philippines who completed a questionnaire reporting the presence and frequency of sexual dysfunction in the past 12 months, the prevalence of selfreported lack of interest in sex was $27 \%$ overall [range $17-$ $50 \%{ }^{26}$ A community-based cross-sectional study in the United Kingdom, Germany, France, and Italy revealed the prevalence of low desire increased from $11 \%$ in women aged 20-29 years to $53 \%$ in women aged $60-70$ years, whereas distress decreased from $65 \%$ to $22 \%$ in the same age groups; therefore, the prevalence of HSDD remained relatively stable in women aged $20-70$ years $(6-13 \%) .{ }^{27}$ In the United States, the prevalence of self-reported decreased sexual desire with distress was $12 \%$ in women aged $45-64$ years, ${ }^{25} 32 \%$ in women aged 40-64 years in Australia, ${ }^{28}$ and $14 \%$ in women aged 65-79 years, also in Australia. ${ }^{29}$

By 2030, using the age of 50 years as a proxy for menopause, the world population of postmenopausal women will be around 1.2 billion, with an incidence of 47 million reaching menopause each year. ${ }^{30}$ In addition, increasing numbers of women experience menopause earlier, with adverse health consequences affecting multiple systems including those relevant to sexual health, often associated with significant distress. The terms premature ovarian insufficiency (less than age 40 years) and early menopause (less than age 45 years) describe the spectrum of conditions associated with the loss of ovarian function before the age of natural menopause. Premature ovarian insufficiency and early menopause include both spontaneous primary ovarian insufficiency (idiopathic; caused by chromosomal and genetic defects; associated with autoimmune disorders, infections, metabolic disturbances, environmental factors) and induced ovarian failure resulting from interventions (pelvic radiation therapy, chemotherapy, surgical oophorectomy including risk-reducing bilateral salpingo-oophorectomy in women with BRCA mutations). ${ }^{31,32}$ Circulating ovarian hormone levels of both estrogens and androgens are abruptly decreased and more substantially reduced after surgical menopause than natural menopause. ${ }^{33,34}$ The prevalence of primary ovarian insufficiency in women younger than 40 years is approximately $1 \%$, younger than 30 years, $0.1 \%$, and younger than 20 years, $0.01 \%$, with little difference by ethnicity. ${ }^{33,35,36}$ Spontaneous early menopause affects approximately another $5 \%$ of women between ages 40 and 45 years. ${ }^{37}$ Although the rate of hysterectomy is declining, a significant number of those women (approximately 40\%) are entering menopause early as a result of hysterectomy with oophorectomy or oophorectomy alone. ${ }^{38-40}$ Thus, there is a considerable need for standardized care to avoid the shortand long-term consequences of premature deprivation and age-related decline of sex hormones. ${ }^{24,32,41}$

\section{Physiology of Androgens in Women}

Testosterone is a metabolic, vascular, and reproductive hormone in women. ${ }^{42-44}$ The production of testosterone in premenopausal women has been estimated to be approximately $0.2-0.25 \mathrm{mg} /$ day. $^{45}$ In premenopausal women, the median circulating testosterone levels are similar to those of estradiol and estrone, ranging from 300 to $400 \mathrm{pmol} / \mathrm{L}{ }^{46}$ Testosterone circulates in nanomolar concentrations in women, whereas estradiol circulates in picomolar concentrations. ${ }^{47}$ Testosterone and its precursors are synthesized by the ovaries and adrenal glands, with about $50 \%$ of circulating testosterone produced by peripheral conversion of these androgen precursors (Figure 1). ${ }^{48}$

Testosterone directly, or via its metabolism to $5 \alpha$ dihydrotestosterone ( $5 \alpha$-DHT) or aromatization to estradiol, modulates many biochemical and physiological pathways, thus regulating cellular metabolism (Figure 2). ${ }^{49,50}$ Testosterone influences sexual differentiation of the genitalia and 


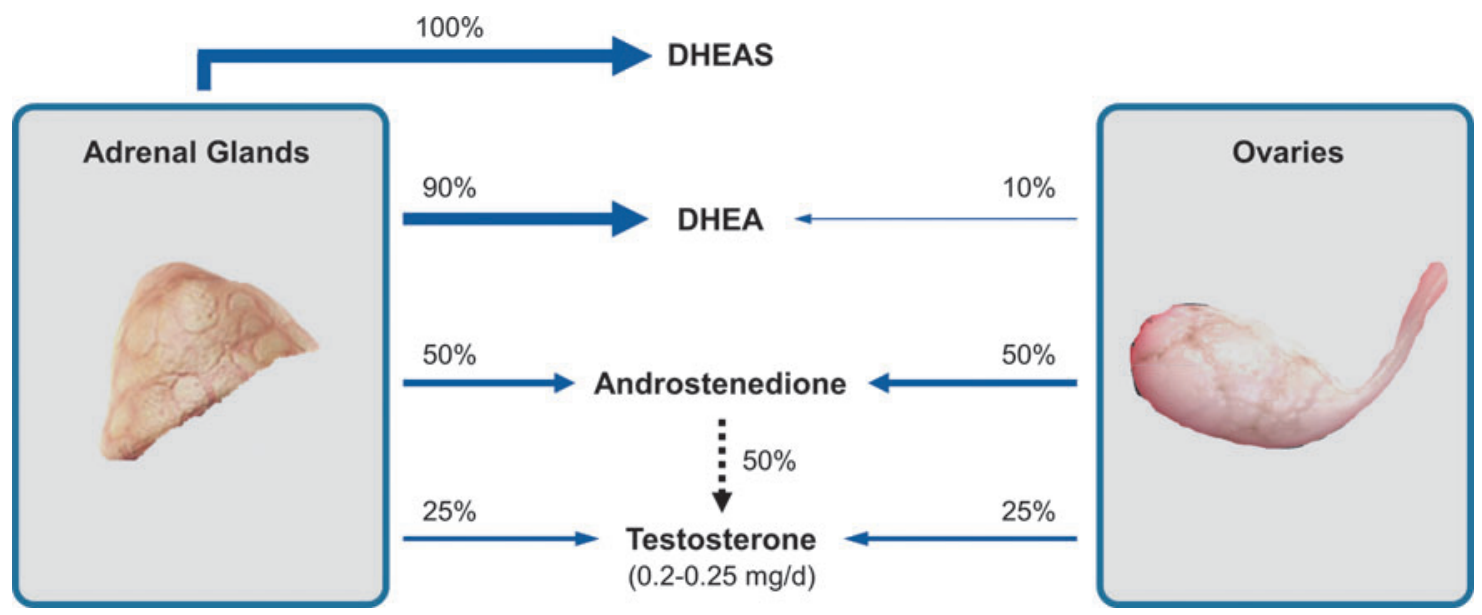

FIG. 1. Relative production of circulating androgens in the adrenal glands and ovaries. The substantial contribution of androstenedione to circulating testosterone is shown by a dashed arrow and involves peripheral tissue conversion. DHEA = dehydroepiandrosterone; DHEA-S = dehydroepiandrosterone sulfate.

the brain, determines secondary sexual characteristics during development and sexual maturation, contributing to maintenance of their functional state in adulthood, and modulates sexual behavior. ${ }^{44} 5 \alpha$-DHT is the most potent androgen and has the highest binding affinity to the androgen receptor (AR), ${ }^{4}$ whereas the testosterone precursors androstenedione and dehydroepiandrosterone (DHEA) are only weakly androgenic. In circulation, testosterone is loosely bound to al- bumin $(\sim 30-45 \%)$ and more strongly bound to SHBG $(\sim 65 \%)$ with a small fraction $(1-3 \%)$ circulating as "free testosterone."

Testosterone and $5 \alpha$-DHT modulate cellular function by genomic and nongenomic mechanisms. ${ }^{51-53}$ The genomic mechanisms involve binding of testosterone or $5 \alpha$-DHT to the AR with concomitant activation and translocation of the ARhormone complex into the nucleus and binding of the complex

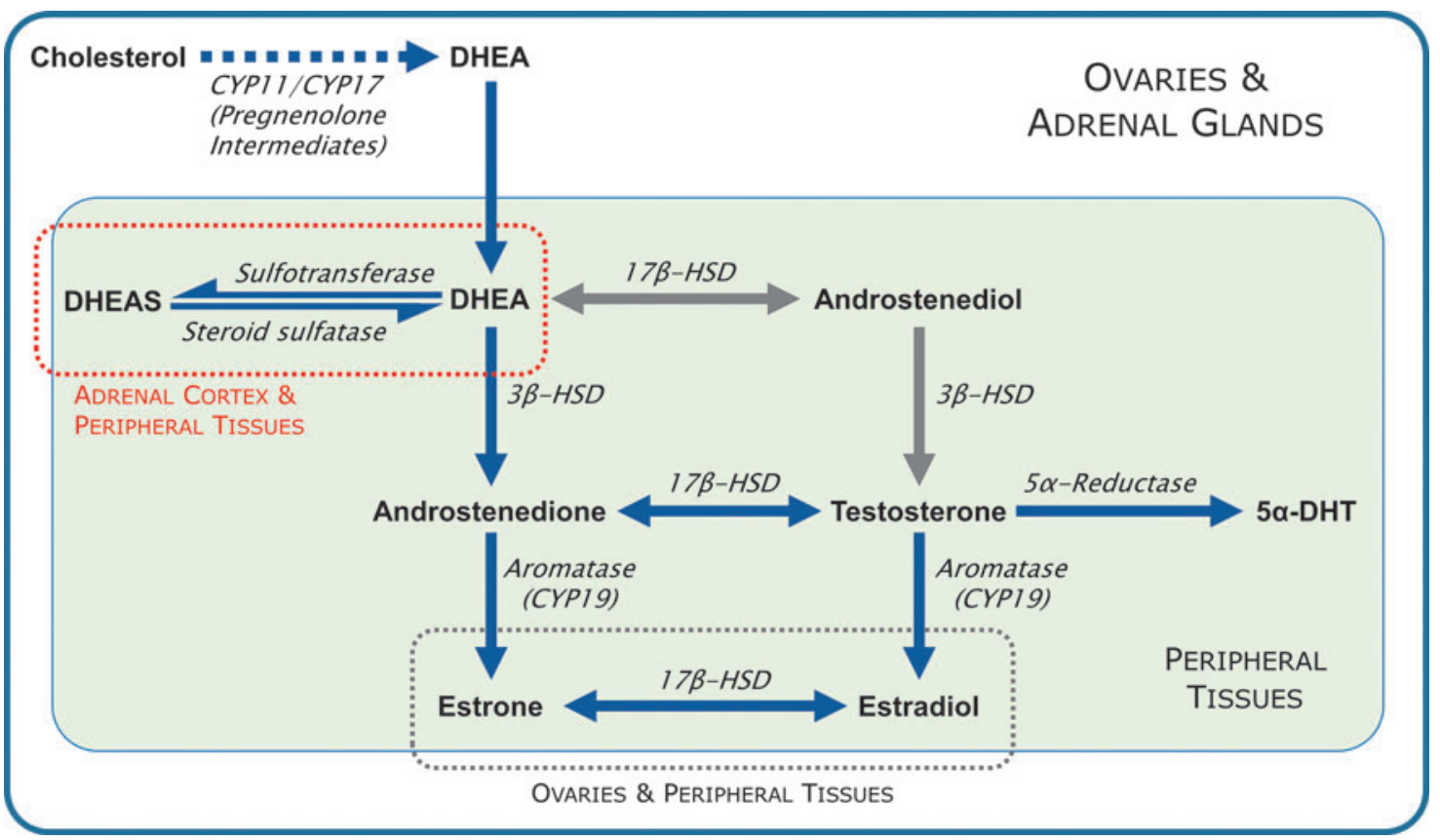

FIG. 2. Synthetic pathways of sex steroids. Intermediate steps involved in the conversion of cholesterol to DHEA are not shown. The ovaries and adrenal glands have a full complement of enzymes to produce androgens and estrogens. In addition, circulating DHEA can be converted to testosterone and estradiol in peripheral tissues (green shaded area). The conversion of DHEA to DHEA-S is limited to the adrenal cortex, whereas DHEA-S can be converted back to DHEA in peripheral tissues (red dotted area). Major pathways of synthesis in humans are denoted by blue arrows, and minor pathways are denoted by gray arrows (adapted from Traish et al). ${ }^{74} \mathrm{CYP}=$ cytochrome $\mathrm{P} 450 ;$ DHEA=dehydroepiandrosterone; DHT= dihydrotestosterone; HSD = hydroxysteroid dehydrogenase. 
to androgen response elements on target genes (Figure 3). This reaction leads to recruitment of transcriptional factors and interaction with receptor coactivators or corepressors resulting in gene activation or repression and modification of cellular metabolism. ${ }^{54,55}$ The nongenomic action of androgens is thought to occur, in part, via binding of testosterone or $5 \alpha$ DHT to a putative membrane AR or to the classical AR. These interactions elicit activation of G-protein-coupled receptors and/or stimulation of intracellular kinases with concomitant increase in the synthesis of second messengers. ${ }^{49,50}$ These nongenomic signaling events may regulate neural activity in the central nervous system, as suggested by human brain imaging studies. ${ }^{56,57}$ However, this has not been studied directly in the context of sexual function. Nevertheless, the wide expression of the AR in various tissues including the female central nervous system and genital and other reproductive tissues suggest that androgens play an important physiological role in female sexual function. ${ }^{58}$

\section{Mechanism of Action of Sex Steroids}

\section{Central mechanisms in animal models}

Regarding the role of testosterone in sexual desire, correlational data from human studies may be more informative than laboratory studies in animals because the assessment of sexual desire in animal studies is limited to monitoring appetitive behaviors. However, observations from animal studies can provide additional insight. In one study of sexually inexperienced female shrews that had low levels of estrogen, testosterone propionate implanted into the medial preoptic area or dorsomedial hypothalamus induced sexual behavior. ${ }^{59}$ Testosterone but not estrogen regulates the activity of specific neurons within the prefrontal cortex that project to dopaminergic neurons in the ventral tegmental area to regulate their burst firing activity in adult rats. ${ }^{60}$ The ventral tegmental area is part of the mesolimbic system that regulates motivational processes and sensitivity to reward. Gonadectomy leads to alterations in hippocampal neuroplasticity that are associated with depressivelike behaviors that can inhibit sexual motivation. ${ }^{61}$ The action of testosterone may also be prolonged by estrogen, as is evidenced by the observation that estradiol increases the duration of AR occupation by $5 \alpha$-DHT in the medial preoptic area, a key brain region regulating sexual behavior. ${ }^{62}$ However, a recent study demonstrates that treating ovariectomized rats with the nonaromatizable androgen $5 \alpha$-DHT enhanced female sexual activity while significantly improving both appetitive and receptive behaviors. ${ }^{63}$ Thus, the function of key brain regions that regulate both motivation and reward are testosterone dependent and mainly mediated by the activation of the AR.

\section{Central mechanisms in women}

The brain is one of the most important targets for sex hormones throughout the life span. Some research supports the role of estradiol in the central modulation of sexual desire. ${ }^{64,65}$ Other research demonstrates that testosterone has enduring influences on many neural and behavioral functions via both genomic and nongenomic actions. ${ }^{66}$ The neuroendocrine mechanisms underlying the influence of testosterone on sexual behavior in women are still not completely understood. It remains unclear whether testosterone positively modulates sexual desire in women through a direct stimulation of the AR or through its conversion to estrogen and subsequent binding to estrogen receptors. ${ }^{67}$ Consistent with preclinical data, 2 lines of evidence point toward a direct, AR-mediated effect of testosterone on sexual behavior in women. One is based on the fact that estrogen-alone therapy in postmenopausal women was associated with a small to moderate improvement in sexual

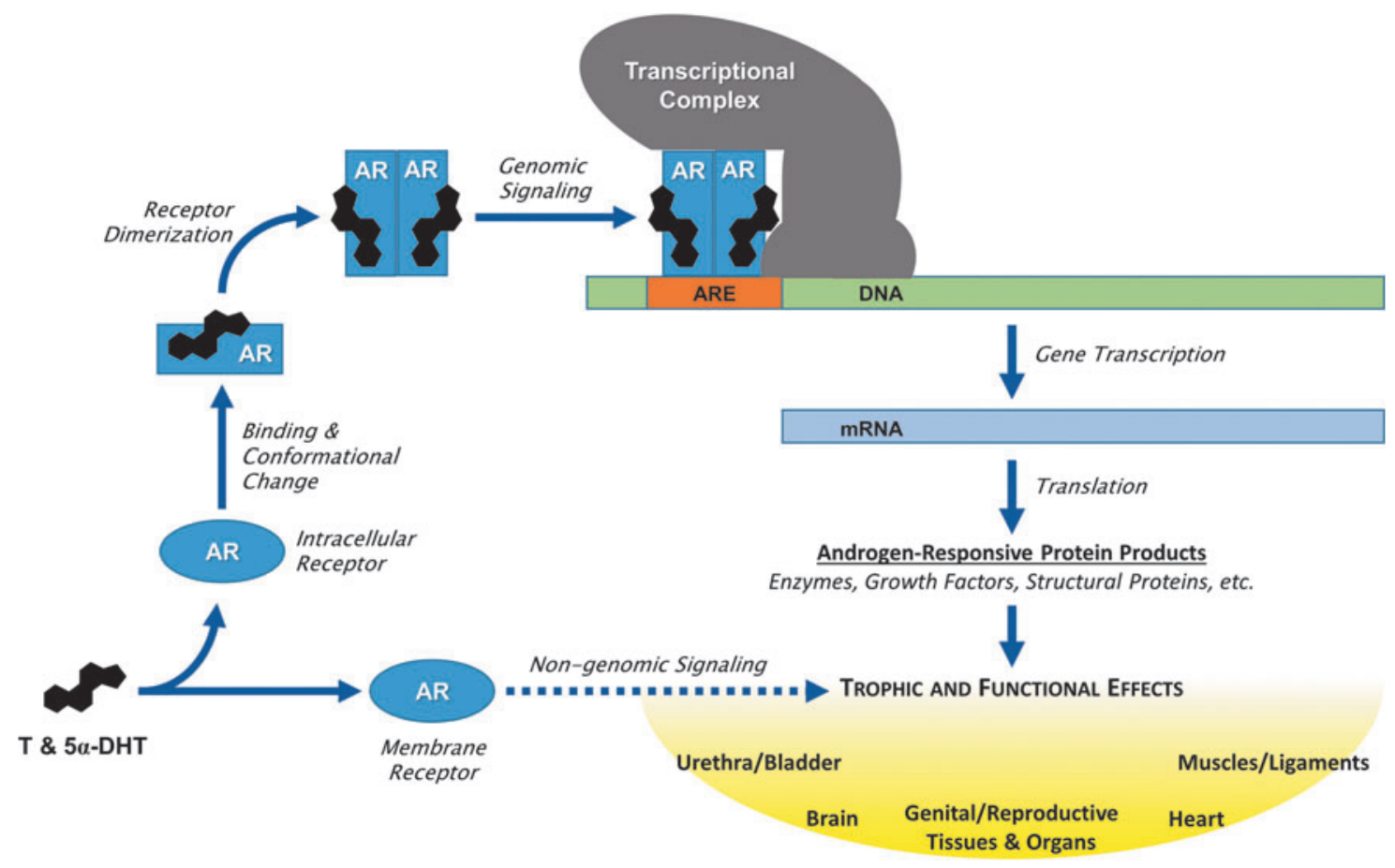

FIG. 3. Androgen receptor (AR) mechanism of action (adapted from Traish et al). ${ }^{74}$ ARE $=$ androgen response element; $\mathrm{DHT}=$ dihydrotestosterone; $\mathrm{T}=$ testosterone. 
function, particularly in pain, with no effect on libido, sexual desire, and interest. ${ }^{68}$ Second, in a study of postmenopausal women using transdermal estradiol and testosterone gel randomized to an aromatase inhibitor or placebo, total and free testosterone in the physiologic range was associated with improvement in sexual satisfaction, well-being, and mood. ${ }^{69} \mathrm{Ar}$ omatase inhibition did not appear to influence the outcome. Existing evidence therefore suggests that testosterone conversion into estradiol is not necessary to exert its beneficial effects on sexuality.

\section{Peripheral mechanisms}

The peripheral responses in female sexual arousal include genital vasocongestion and vaginal lubrication, which result from increased blood flow to the clitoris, vulva, labia, and vagina. $^{70,71}$ The basic mechanisms of these hemodynamic processes are mainly adrenergic and nitric oxide signaling, although modulation by sex steroid hormones is also involved. ${ }^{58,72}$ Androgens and estrogens regulate the structure and function of genital organs and modify the female genital sexual arousal response in women by the following mechanisms ${ }^{58,73}$ : (1) regulation of sex steroid receptors, (2) maintenance of genital tissue structures and function, and (3) modulation of vaginal lubrication.

Regulation. The ARs have been detected throughout genitourinary tissues, such as the vagina, clitoris, labia, vestibule, bladder, and supporting structures of the pelvic floor. ${ }^{74-76}$ Testosterone and estradiol regulate their own receptors and can also cross-regulate the other receptors. ${ }^{74}$ Testosterone is metabolized to $5 \alpha$-DHT in peripheral tissue.

Maintenance. Estradiol modulates genital hemodynamics and is related to the density of collagen fibers in the lamina propria and moderates muscular thickness in the vagina and vasorelaxant mechanisms of the clitoris. ${ }^{77,78}$ Androgens maintain nonvascular smooth muscle function in the vagina and vascular smooth muscle in the clitoris and also have a stimulatory effect on the vaginal nerve density. ${ }^{58,77,79}$

Modulation. Vaginal lubrication is a combination of basal mucin production and vaginal vascular transudate. ${ }^{79}$ Sex steroid hormones regulate mucification, keratinization, and permeability of the vaginal epithelium. ${ }^{58}$ Mucification of the vaginal epithelium is an androgenic effect, and proliferation of the vaginal epithelium is an estrogenic effect. ${ }^{77,79}$ Therefore, mucin production is regulated by androgen, whereas vaginal vascular transudation is an estrogen-dependent effect.

Androgens play an important role in genital sexual response. This guideline will address the impact of and indications for systemic testosterone therapy and will not focus on intravaginal androgens.

\section{Role of Laboratory Evaluation of Androgen Levels in Managing HSDD}

\section{How is testosterone measured?}

Direct assays measure total testosterone in women using the radioimmunoassay (RIA) technique. Assays have not been standardized for this purpose at either a national or international level, and total testosterone concentrations may be reported as being substantially different for a single sample by different laboratories. Hence, direct assays for the measurement of total and free testosterone are highly unreliable in the female range.

Total testosterone can be measured with high accuracy and reproducibility using liquid chromatography (LC)/gas chromatography (GC) and LC-tandem mass spectrometry (MS/MS) assays. ${ }^{6,80}$ However, it is important to note that LC-GC and LC-MS/MS have also not been internationally standardized. If neither LC-GC nor LC-MS/MS is available, measurement of total testosterone using direct assays routinely used in clinical laboratories is appropriate.

\section{What is the association between androgen levels and aging?}

Androgen levels decline with age and drop abruptly after bilateral oophorectomy. ${ }^{47}$ The decline in androgen levels in postmenopausal women appears to be a function of age, aging ovarian function, and decrease in adrenal precursor steroids. ${ }^{81}$ Cross-sectional studies of healthy women demonstrate a substantial decrease in androgen levels across the reproductive years with no significant change across the menopausal transition (ages 45-54 years). ${ }^{42,46}$ In one study, women who had undergone bilateral oophorectomy (aged 55 years or older), not on exogenous steroids, had 40-50\% less serum total and free testosterone concentrations measured by the RIA compared with age-matched naturally menopausal women. ${ }^{42}$ A recent cross-sectional study that measured sex steroids by LC-MS/MS demonstrated that in women older than 70 years, serum concentrations of DHEA (a testosterone precursor) are lower than in premenopausal women and continue to decline with age, but total testosterone levels are similar to those seen in premenopausal women and appear to increase slightly beyond the age of 70 years. ${ }^{82}$

\section{Do androgen levels correlate with sexual function and predict HSDD?}

Research determining the associations between the androgens (testosterone and its metabolite $5 \alpha$-DHT) has been limited by the low levels in women, insufficient sensitivity and specificity of immunoassays to evaluate these low levels and, until recently, a high degree of cross-reactivity between testosterone and other circulating steroids. The limited available data indicate weak, independent associations between testosterone (measured by LC-MS/MS) with sexual desire, orgasm, and self-image in premenopausal women ${ }^{83,84}$ and testosterone (measured by the RIA) with sexual desire, arousal, and masturbation frequency in midlife women. ${ }^{85}$ Early follicular phase levels of total and free testosterone, androstenedione, and dehydroepiandrosterone sulfate (DHEA-S) have been positively correlated with self-reported sexual desire in premenopausal women aged 25-44 years not using hormonal contraception, ${ }^{83}$ whereas no correlation was found in women with HSDD. ${ }^{86}$ A recent study of women aged 18-39 years, with regular menstrual cycles, not using any sex steroid therapy who had sex steroids measured by LC-MS/MS, found weak positive associations between both sexual desire and sexual pleasure and DHEA and androstenedione. ${ }^{84}$ Orgasm was positively correlated with both testosterone and androstenedione. All analyses were adjusted for age, body mass index, cycle stage, smoking, parity, partner status, and psychoactive medication. 
In a study examining HSDD in postmenopausal women, the prevalence was significantly greater in surgically menopausal women aged 20-49 years (26\%) than age-matched premenopausal women $(14 \%){ }^{87}$ There was no significant difference in the prevalence of HSDD between surgically and naturally menopausal women aged 50-70 years. These different rates have been attributed to the abrupt loss of ovarian androgen and significantly greater personal distress in young surgically menopausal women, indicating that testosterone may contribute substantially to sexual desire. ${ }^{87}$

A large epidemiological investigation found a poor correlation between any steroid hormone measured by immunoassay and low sexual desire or function. ${ }^{88}$ Although decreased levels of DHEA-S less than the tenth percentile had the best correlation with low sexual responsiveness in older women aged 45-75 years, most women with low DHEA-S did not have any sexual dysfunction. ${ }^{88}$ Thus, there is no absolute level of any androgen that signals HSDD.

The lack of clear correlation between sexual function and declining androgen levels has been explained by the complexity of androgen metabolism and measurement ${ }^{88}$ and the observation that circulating testosterone levels do not adequately reflect tissue concentrations. ${ }^{47,89}$ Although circulating hormone levels have been used as indicators of tissue exposure, a significant proportion of androgen synthesis may be intracrine, such that circulating precursors and testosterone act as prohormones and are converted to active hormones within target cells. The complex regulation of AR expression plays a key role in androgen effects. Furthermore, androgenic effects vary according to individual variations in the amount and activity of the enzymes $5 \alpha$-reductase and aromatase and individual differences in the AR response. ${ }^{89,90}$ However, it is still not clear whether the AR activity is a modulator of the androgenic effect on sexual desire ${ }^{90}$ or if levels of intracrine activity of androgens are better parameters for androgen effect on sexual desire. ${ }^{83}$

\section{What is the rationale for testosterone therapy in women?}

Although no testosterone serum concentration correlates with the presence or absence of HSDD or its severity, ${ }^{88}$ there is a loose correlation between testosterone concentration while undergoing treatment and its benefits for improving sexual dysfunction. ${ }^{47}$ Although the complexity of central and peripheral mechanisms of action of testosterone on sexual desire is not completely understood, research and clinical evidence support a positive effect of testosterone therapy on sexual desire when premenopausal physiologic levels are maintained. ${ }^{47}$ When testosterone supplementation exceeds the normal premenopausal range, which is the recommended optimal treatment threshold, sexual desire may actually decrease, suggesting a bimodal effect. ${ }^{91}$

\section{Are there normal values and treatment targets?}

As testosterone is measured by a variety of methods with different reference standards, it is not possible to establish a universal reference range for premenopausal women. Historically, the adequacy of serum testosterone therapy targeting premenopausal values was estimated using the free androgen index (FAI) with assessment of morning total testosterone (T) and SHBG. ${ }^{92}$ These values were "plugged into" the equation to calculate the FAI as follows: FAI $=100 \times[$ Total T $(\mathrm{ng} / \mathrm{dL})] /$ SHBG (nmol/l)]. ${ }^{93}$ The concentration of free or bioavailable testosterone was approximated, but this approach has limitations as it assumes predictable binding of testosterone to SHBG at all concentrations. ${ }^{92,93}$

An attempt to extend this method for determination of a discriminatory level correlated to sexual function and dysfunction was published by Guay et al. in 2004. ${ }^{92}$ They studied 60 healthy, menstruating women not on hormonal contraception, ages 20-49 years, without sexual dysfunction as assessed by a validated questionnaire and detailed interview. RIA measurements of morning testosterone, SHBG, and multiple other androgens were assayed; the FAI was calculated. Based on this small cohort, these investigators proposed a clinically useful range of the normal androgen profile in women without sexual dysfunction.

The applicability of this method in clinical practice is limited given today's alternatives for several reasons: (1) the small sample sizes of the original publications, (2) insufficient accuracy and precision of RIA testosterone measurements, ${ }^{94,95}$ (3) the availability of better methodology for clinical use, and (4) an improved understanding of the binding characteristics of testosterone in serum across the entire range spectrum. ${ }^{96}$

\section{Why should testosterone be measured?}

Although there are limitations with total testosterone measurement in clinical practice, the Global Position Statement and this consensus panel recommend total testosterone as the best available measure, rather than free or bioavailable testosterone or the FAI. Therapy should not target total testosterone concentrations that exceed those of premenopausal women. There is no definitive testosterone cutoff level to identify women with sexual dysfunction including HSDD. ${ }^{6}$ There is no blood level that is a treatment goal for testosterone therapy, as serum concentrations do not predict treatment efficacy. The main reasons to measure testosterone are (1) to exclude women with midrange to high values (according to the assay used) that would suggest against androgen levels being associated with the patient's symptoms and (2) to monitor testosterone therapy to ensure against supraphysiological values and associated androgen excess side effects.

\section{Clinical Trial Data Regarding Efficacy of Testosterone}

There is consistent evidence from placebo-controlled randomized clinical trials (RCTs) that testosterone therapy is more effective than placebo for the treatment of HSDD in postmenopausal women. $6,97,98$ The earlier studies evaluated testosterone implants 99,100 and oral testosterone formulations ${ }^{101,102}$; however, these modes of testosterone administration often result in supraphysiological concentrations. ${ }^{100,103}$ In contrast, treatment of women with transdermal testosterone as a patch releasing $300 \mathrm{mcg}$ of testosterone/day ${ }^{104}$ or a manufactured cream delivering $5 \mathrm{mg}$ testosterone in $0.5 \mathrm{~mL}$ $(10 \mathrm{mg} / \mathrm{mL})^{105}$ daily results in free testosterone levels within the normal premenopausal range. Transdermal testosterone improves sexual desire and reduces sexually associated personal distress in naturally and surgically menopausal women, with and without concurrent estrogen \pm progestogen therapy, 
presenting with HSDD. ${ }^{5,98,104}$ In studies of women with HSDD, transdermal testosterone also improved the frequency of satisfying sexual events, arousal, orgasm frequency, pleasure, responsiveness, and self-image while reducing sexual concerns. ${ }^{5}$ In 2 studies, surgically menopausal women with HSDD treated with transdermal testosterone experienced a statistically significantly greater meaningful treatment benefit than those who received placebo, as reported during face-to-face interviews. Women who identified with having clinically meaningful benefit also achieved larger statistically significant improvements in sexual desire, satisfying sexual activity, and personal distress than those who did not achieve clinically meaningful benefit from the therapy. ${ }^{106}$

The data for the effects of testosterone in premenopausal women are limited to women in the late reproductive years. Although the 3 placebo-controlled RCTs of testosterone in premenopausal women ${ }^{107-109}$ suggest favorable effects of transdermal testosterone on sexual function in older premenopausal women presenting with low sexual desire, the numbers of women in each study were small. A recent meta-analysis demonstrated the need for further research to determine the efficacy of testosterone therapy for premenopausal women with HSDD. ${ }^{5}$

\section{Clinical Trials Data Regarding Safety of Testosterone}

\section{Short-term safety}

Testosterone preparations, specifically IM, subcutaneous implants, and oral formulations (methyltestosterone 1.25$2.5 \mathrm{mg}$ or testosterone undecanoate $40 \mathrm{mg}$ ), often in combination with estrogen therapies, have been evaluated in RCTs with relatively few participants and followed for relatively short periods of time. The longest duration placebo-controlled trial (24 months) included 331 subjects. ${ }^{110,111}$ These preparations are not commonly used currently. IM administration is associated with wide excursions of serum testosterone concentrations well beyond physiological levels; subcutaneous implants occasionally yield markedly elevated testosterone levels with erratic release over time and difficulty locating for removal if necessary. Oral testosterone undecanoate was associated with unpredictable absorption and blood levels in the male range, even at the lowest dose studied. ${ }^{103}$

In a meta-analysis of 36 trials enrolling 8,480 women with duration of 12 weeks-2 years, testosterone administration (oral preparations in approximately half the trials; IM injection, subcutaneous pellets, and transdermal preparations in the remainder) increased the risk of developing acne (relative risk 1.46; 95\% confidence interval [CI] 1.11-1.92) and hair growth (relative risk 1.69; 95\% CI $1.33-2.14$ ). ${ }^{5}$ Findings were comparable with a prior meta-analysis. ${ }^{111}$ No significant improvement was noted for bone mineral density, body composition, or cognitive measures, although an overall increase in weight was recorded with testosterone treatment. ${ }^{5}$ Lipid effects included reduction in total cholesterol, highdensity lipoprotein cholesterol, and triglyceride levels along with a statistically significant increase in low-density lipoprotein cholesterol with oral but not with transdermal testosterone preparations. ${ }^{5,97,111}$ Also, these older oral formulations are no longer recommended because of concerns with negative hepatic effects such as peliosis hepatis and hepatic neoplasms including hepatocellular carcinoma. ${ }^{112,113}$
Most of clinical investigations in the past 2 decades have focused on transdermal testosterone patches with release rates of $150 \mathrm{mcg}$ and $300 \mathrm{mcg} / 24 \mathrm{~h}$. Accordingly, a metaanalysis of safety outcomes and adverse events reported from 7 trials, enrolling 3,035 participants, randomized to transdermal testosterone $300 \mathrm{mcg}$ or placebo, either on or not on estrogen-progestin hormone therapy and followed for 24 weeks found that total androgenic adverse events were increased (hazard ratio 1.37; 95\% CI, 1.12-1.69), including acne (hazard ratio $1.41 ; 95 \% \mathrm{CI}, 1.05-1.88$ ) and hair growth (hazard ratio 1.56; 95\% CI, 1.17-2.09), without evidence of alopecia, clitoromegaly, or voice deepening. ${ }^{98}$ Serum lipid profiles, carbohydrate metabolism, cardiometabolic markers, and renal and liver functions, were similar among treatment and control groups. ${ }^{5,98}$ Mammographic breast density did not change with transdermal testosterone treatment in several small trials with insufficient data to assess long-term breast cancer risk. ${ }^{5,6}$ Although data are limited, there were no significant differences in total adverse events, serious adverse events, or withdrawal rates from the studies. ${ }^{5}$ Safety data from RCTs using testosterone in physiologic doses are not available beyond 24 months of treatment. ${ }^{6}$

\section{Long-term safety}

One 4-year open-label extension of 2 6-month clinical trials of $300 \mathrm{mcg} /$ day transdermal testosterone patch in 967 women with oophorectomy treated with concomitant estrogen therapy showed no increase in the rate of serious adverse events. ${ }^{114}$ A phase 3 cardiovascular and breast safety RCT of $1 \%$ testosterone transdermal gel, $300 \mathrm{mcg}$ daily was undertaken in 2008 and enrolled 3,656 postmenopausal participants older than 50 years, with at least 2 cardiovascular risk factors at baseline (e.g., hypertension, dyslipidemia) and a clinical diagnosis of HSDD. ${ }^{115}$ The composite cardiovascular end point included death, nonfatal myocardial infarction, nonfatal stroke, coronary revascularization, hospitalized unstable angina, and venous thromboembolic events. ${ }^{115}$ After 4 years, with more than 7,300 women-years of exposure, the company (BioSante Pharmaceuticals, Inc.) reported 53 adjudicated cardiovascular events, a lower than anticipated rate, with the number of breast cancers as anticipated based on subject ages. ${ }^{116}$ Study results have been published in press releases but not in peer-reviewed publications. Despite these reassuring outcomes, long-term (beyond 2 years) safety data, particularly regarding breast cancer and cardiovascular events (acute myocardial infarction, stroke, deep vein thrombosis, and death), are limited and inconclusive. ${ }^{5,6,15,117-120}$

\section{Future strategies to assess safety}

Several recent meta-analyses and the 4-year open-label extension trial provide safety reassurance for the use of transdermal testosterone therapy in postmenopausal women with doses that approximate physiological levels for premenopausal women. ${ }^{114}$ However, reported RCTs to date have neither been large enough nor long enough to definitively determine the effects of testosterone therapy on safety outcomes of interest: cardiovascular events, breast cancer risk, endometrial and ovarian effects, cognitive health, mood, and musculoskeletal health. 
One interim approach would be to establish longitudinal observational studies and patient registries to supplement completed RCTs. It would be clinically useful to stratify safety data by menopausal status, ovarian status (intact vs oophorectomized), and concurrent estrogen and progestogen therapies. As with studies of estrogen and progestin therapies, reassurances of safety would be strengthened with stratification of cardiovascular outcomes (venous thromboembolic events, stroke, myocardial infarction), by age, time since menopause, and metabolic characteristics to facilitate advising women at different baseline risk.

\section{Management Guidelines}

A summary of the key clinical recommendations for this Clinical Practice Guideline is described in Table 1. The levels of evidence supporting these key points are discussed in the Global Position Statement. ${ }^{6}$ Where evidence is not available, this panel relied on expert opinion and widely established clinical principle. ${ }^{19}$

\section{Diagnosis of HSDD}

Following the ISSWSH Process of Care (POC) for HSDD, assessment of low sexual desire includes the use of the validated self-report screening and diagnostic instrument, the Decreased Sexual Desire Screener, ${ }^{121}$ that enables the diagnosis of generalized, acquired HSDD and identification of associated biopsychosocial factors. The clinician and the patient should discuss the results of the Decreased Sexual Desire Screener to determine the relative importance of these contributors. Recommended strategies also include a detailed

\section{Table 1. Key Take-Away Messages}

- Androgens, including testosterone, are essential hormones for development and maintenance of female sexual anatomy and physiology and modulation of sexual behavior.

- Testosterone has many physiological actions in women, directly through its cell-specific receptor, by nonreceptor-mediated actions, and by conversion to $5 \alpha$-DHT and estrogens.

- There is no testosterone level for diagnosis of HSDD or for use as a treatment target.

- Total testosterone concentration is the best practical assay.

- Total testosterone and SHBG should be measured before initiating therapy.

- Proper dosing should attain and maintain total testosterone levels in the premenopausal physiological range.

- If an approved female formulation is not available, onetenth of a standard male dose of $1 \%$ transdermal testosterone or about $300 \mathrm{mcg} /$ day can usually achieve the normal premenopausal physiological range.

- Compounded testosterone, pellets, IM injections, and oral formulations are not recommended.

- Additional testing and alternative strategies may be required to assess failure to respond to typical testosterone treatment, particularly when testosterone or SHBG levels are high.

$5 \alpha$-DHT $=5 \alpha$-dihydrotestosterone; HSDD $=$ hypoactive sexual desire disorder. sexual history that incorporates information about sexual desire, arousal, orgasm, and pain and other aspects of sexual activity and function as indicated. ${ }^{15}$ Sexual history taking may involve partnered and unpartnered sexual activity and past and current partner relationships and sexual experiences. Regarding discrepancy of desire between the patient and her partner, the patient should be diagnosed with HSDD only if the discrepancy causes her distress. ${ }^{15}$

Confounders of normal desire such as concomitant medications (e.g., antidepressants, antihypertensives), life events (e.g., pregnancy, lactation, surgery), and relationship issues should be fully explored. In women diagnosed with generalized, acquired HSDD, a complete physical and gynecological examination when appropriate and laboratory testing (in addition to testosterone and SHBG) when indicated (prolactin, thyroid function panel, estradiol, progesterone, luteinizing hormone) are recommended to ascertain any modifiable factors. ${ }^{15}$

\section{Biopsychosocial treatment model}

Female sexual dysfunctions such as HSDD have multiple etiologies representing biopsychosocial factors including neuroendocrine imbalance, physical health, disease, interpersonal difficulties, psychological distress, and sexually repressive cultural and religious values. ${ }^{6,122}$ The ISSWSH POC for HSDD provides an efficient way to diagnose HSDD and to identify etiologic factors and a subsequent treatment pathway. 6,15

Treatments should follow a biopsychosocial model and include pharmacologic options (hormonal and nonhormonal agents), psychotherapy, or multimodal treatments that combine pharmacologic with nonpharmacologic treatments. The optimal approach includes identifying which biopsychosocial factor(s) are compromised, likely contributing to distressing symptoms, and amenable to evidence-based solutions that are tailored to the predisposing factors. ${ }^{15,122}$ If psychosocial factors have been modified, or if these factors are present but do not appear to be the primary source of the onset of sexual dysfunction, or if there are no psychosocial factors, biological etiologic factors should be considered. Systemic testosterone targeting central nervous system and neuroendocrine mechanisms have clear efficacy for the treatment of HSDD in postmenopausal women. ${ }^{15}$ Even when the primary etiology is biological, symptoms may be maintained or exacerbated by psychosocial and interpersonal factors that have been the consequence of a biologically based female sexual dysfunction. Furthermore, when the etiology of generalized, acquired HSDD appears to be multifactorial without a predominant factor identified, pharmacologic treatment can be considered. Combination therapy (psychological and pharmacologic therapy) may be beneficial in all of these circumstances.

\section{When to treat}

Testosterone therapy should only be initiated after a full biopsychosocial evaluation and appropriate management of other conditions that may contribute to diminished desire such as dyspareunia, fatigue secondary to vasomotor symptoms, anemia, thyroid disease, anxiety and depression, medication side effects, and relationship issues. ${ }^{15}$ The presence of these conditions does not preclude commencing 
testosterone as long as the identified conditions are being concurrently addressed. As discussed previously, psychological and pharmacologic therapy may be combined when appropriate.

\section{Who to treat}

Candidates for testosterone therapy are postmenopausal women presenting with a decline in sexual interest with or without diminished arousal that causes sufficient personal or interpersonal concern (distress) that they seek treatment. Women should not receive testosterone therapy if they have signs of clinical androgen excess (i.e., acne, hirsutism, androgenic alopecia) or are using an antiandrogenic medication (e.g., finasteride, dutasteride). Testosterone treatment in women with hormone-dependent neoplasia should only be recommended in consultation with the specialist(s) providing the cancer care. Women with a high SHBG concentration are less likely to experience treatment benefit. ${ }^{123}$

Although the Global Position Statement endorses testosterone therapy only for postmenopausal women, the ISSWSH POC for HSDD includes women in the late reproductive years, a recommendation supported by the physiology of decline in androgens and the efficacy data discussed previously. 6,15

\section{Special populations}

Antidepressant treatment-emergent sexual dysfunction. In the only RCT of women $(n=44)$, primarily premenopausal, experiencing selective serotonin reuptake inhibitor or serotonin-norepinephrine reuptake inhibitor treatment-emergent loss of libido, transdermal testosterone increased the frequency of satisfying sexual events but did not show statistically significant changes on measures of sexual function and sexual distress. ${ }^{108}$ Therefore, the data are insufficient to recommend testosterone for treatment-emergent sexual dysfunction from selective serotonin reuptake inhibitors and serotonin-norepinephrine reuptake inhibitors.

Premature ovarian insufficiency and early menopause. Menopausal hormone therapy (MHT) is strongly recommended for women with early menopause, at least until the age of natural menopause, for relieving symptoms and preventing long-term medical conditions. ${ }^{124,125}$ Physiological replacement of estrogen (and progesterone if the uterus is intact) is essential for health and quality of life. The controversies surrounding the use of MHT concerning benefits and risks should not apply to the premature and early menopause population. ${ }^{126-129}$ Although there is evidence supporting the use of estrogens with adequate endometrial protection with progestogens (when the uterus is present) in women with premature and early menopause, there is a lack of evidence supporting the use of MHT for management of sexual dysfunction in this population. ${ }^{130}$ The guiding principles are to avoid decreasing testosterone by using transdermal estradiol rather than oral estrogens because transdermal preparations exert minimal effects on SHBG and free testosterone levels ${ }^{131}$ and modest improvement in sexual function of early postmenopausal women. ${ }^{132}$ Another approach to maintaining testosterone levels is the use of a bioidentical (biologically identical to the hormones produced in vivo) estradiol containing contraceptive pill (estradiol valerate and dienogest, estradiol and nomegestrol acetate) in late reproductive age women requiring contraception, taking into account the androgenicity of some synthetic progestogens. ${ }^{133,134}$ The Fourth International Consultation of Sexual Medicine recommended conducting studies of testosterone therapy for women with premature ovarian insufficiency presenting with desire and other sexual problems. ${ }^{73}$ Until data are available to guide clinical care, expert opinion and clinical principle indicate that assessment and management of these women should be the same as for any other postmenopausal woman presenting with HSDD. ${ }^{32,73}$ Although the chance of pregnancy is extremely rare in these younger women, testosterone is not recommended for women who are or may become pregnant, as masculinization of a female fetus can occur although only with a very hyperandrogenic state. ${ }^{135}$ Women with premature and early surgically induced menopause are a particularly important cohort for consideration of testosterone therapy because of their experience of abrupt loss of ovarian androgen and the substantial prevalence of HSDD. ${ }^{83,87,136}$ This population was included in RCTs of testosterone therapy in postmenopausal women aged 24-70 years. ${ }^{11,12,136}$ Results demonstrated improvement in sexual function including desire; however, age analyses were not performed.

\section{Formulation}

Despite data supporting the efficacy of transdermal testosterone for the treatment of HSDD without evidence of serious adverse effects, testosterone formulations that deliver a therapeutic dose appropriate for women have generally not been approved by national drug regulatory authorities. When implementing testosterone treatment for postmenopausal women with HSDD, and an approved female formulation is not available, it is reasonable to prescribe off-label an approved male formulation ${ }^{6}$ at approximately one-tenth of the male dose. Compounded products lack evidence for efficacy and safety and may have variability in testosterone concentration leading to concerns about quality. In concurrence with the recent report from the National Academies of Science, Engineering, and Medicine, compounded products cannot be recommended for the treatment of HSDD. ${ }^{6,137,138}$

Testosterone products for men are government inspected for manufacturing quality. If a compounded product is used, the compounding pharmacy should meet industry standards for purity of Active Pharmaceutical Ingredients and Good Manufacturing Practice. ${ }^{6}$

Historically, there have been some formulations approved for women. Testosterone implants (Testosterone Implant, Organon) were previously approved for use in Australia but are no longer available. Oral methyltestosterone, combined with conjugated equine estrogens or esterified estrogens, were never formally approved for treatment of sexual dysfunction in postmenopausal women in the United States. These formulations were not specifically evaluated for HSDD, and the conjugated equine estrogens with methyltestosterone product is no longer available. The transdermal testosterone patch (Intrinsa), developed for treatment of HSDD, was approved in Europe but only for the treatment of surgically menopausal women with HSDD with adequate concomitant estrogen therapy. As the European Medicines Agency refused to broaden the treatment indication to 
naturally menopausal women, the product was withdrawn from the market. A transdermal 1\% testosterone cream (AndroFeme, Lawley Pharmaceuticals, Perth, Australia) is available by prescription in Australia, approved in 2020 by the Australian Register of Therapeutics Goods for treatment of HSDD in postmenopausal women.

Transdermal treatment provides the most physiological form of replacement therapy for women administered as a patch, ${ }^{98}$ gel, ${ }^{69,139}$ cream, ${ }^{105}$ or spray. ${ }^{109}$ Although testosterone implants have been widely used, they may result in supraphysiological levels, do not allow for dose titration, and therefore are not recommended. ${ }^{99,100,103,138}$ Owing to the adverse effects related to short-term safety discussed previously, IM injections and oral preparations are not recommended. Compounded buccal lozenges and troches will result in substantial gut absorption through swallowing of saliva as they are held in the mouth and thus should be considered to be oral testosterone therapy.

\section{Dosing}

Before prescribing testosterone therapy, clinicians should obtain informed consent. They should explain that for women it is an off-label therapy, counsel regarding the benefits and risks through shared decision-making recognizing patient's goals and concerns, and provide detailed documentation of the discussion. ${ }^{140,141}$

Dosing should be targeted to achieve testosterone concentrations in the physiologic premenopausal range. In a study of premenopausal women with no complaints of sexual dysfunction intended to define normal female androgen values, women aged 20-29 years, 30-39 years, and 40-49 years had testosterone values ranging from 45.5 to $57.5 \mathrm{ng} / \mathrm{dl}, 27.6$ to $39.8 \mathrm{ng} / \mathrm{dl}$, and 27.0 to $38.6 \mathrm{ng} / \mathrm{dl}$, respectively. ${ }^{142}$ This range is approximately $1 / 10$ th $-1 / 15$ th of the systemic testosterone values in healthy men (range $300-800 \mathrm{ng} / \mathrm{dl}$ ). ${ }^{143}$ Thus, treatment using any male product for women should be initiated using one-tenth of the recommended starting dose for men.

Men with hypogonadism prescribed daily $1 \%$ generic testosterone gel use 30 tubes or packets per month; the starting dose for women with HSDD should be one-tenth of a tube or packet each day or 3 tubes per month. Resealable tubes are much preferred to packets to avoid evaporation and resulting change in testosterone concentration in the gel; tubes should be specified when prescribing testosterone gel. Using resealable tubes at room temperature, one-tenth of a male dose is approximately 4 drops/day. With the $1 \%$ cream available in Australia, the recommended starting dose is $5 \mathrm{mg}$ /day testosterone $(0.5 \mathrm{ml})$, which may be titrated to $10 \mathrm{mg}$ /day $(1.0 \mathrm{ml})$ as needed. ${ }^{105}$

These topical preparations should be applied to a skin surface, typically the back of the calf or the upper outer thigh (at least an hour after shaving) or the buttock. The patient should be counseled about potential transference of testosterone from the application site to the skin of individuals (young children, female partners) and pets with whom the woman has close contact, while the risk of exposure to a male partner is minimal. ${ }^{144}$ The FDA warnings for men should be explained to women including the need to wash hands immediately with soap and water after application of gel. If contact does occur, the residual testosterone can be washed off with soap and water. Long-term continual exposure may result in signs of virilization, changes in body hair, and acne although these adverse effects are described in case reports of transference from men to women and children. ${ }^{144}$

Patients should be monitored for clinical response to treatment, including increase in sexual desire and decrease in personal distress. ${ }^{140}$ Based on clinical trial data, they should be counseled that on average efficacy emerges 6-8 weeks after initiation of therapy, but many women feel improvement after 4 weeks. ${ }^{123}$ Maximal effects in sexual desire and satisfactory sexual events occur at about 12 weeks. ${ }^{109,123}$ Reductions in sexually associated personal distress are seen at about 4 weeks, and distress continues to decline over the following 5-6 months of therapy. ${ }^{123}$

Although the self-report validated instruments that assess outcomes similar to those used in clinical trials (e.g., Female Sexual Function Index, Female Sexual Distress ScaleRevised) ${ }^{145,146}$ can be administered to assess sexual desire and associated distress, clinical response is generally based on the clinician and patient dialog and conclusions regarding subjective, qualitative improvements.

If a woman does not experience clinically meaningful improvement, treatment should not be continued beyond 6 months ${ }^{6,73,117}$ and other causes for the symptoms should be reinvestigated. In women not responding to testosterone therapy, measurement of $5 \alpha$-reductase could be considered, as some experts propose that women with reduced $5 \alpha-$ reductase activity may not respond to physiologic doses of transdermal testosterone. ${ }^{147}$ If testosterone therapy results in improvement of HSDD, the expert panel recommended that the woman continue for 6-12 months and then consider taking a drug holiday to see if treatment is still required. Furthermore, the panel concluded that although there is a lack of long-term safety data, ongoing testosterone therapy may be needed to maintain the improvement in HSDD.

\section{Monitoring}

Total testosterone levels should be measured before initiating therapy to exclude women with midrange to high baseline testosterone concentrations. SHBG should also be measured as women with levels greater than the normal range are less likely to benefit from therapy. ${ }^{148}$ High SHBG can be seen in healthy women but is also associated with the use of oral estrogens, including estrogen-containing hormonal contraceptives, thyroid replacement, ${ }^{149,150}$ and untreated hyperthyroidism. ${ }^{151}$ In women with high SHBG with unmodifiable factors (e.g., essential thyroid replacement), a trial of testosterone therapy is still worthwhile.

Total testosterone levels should be assessed 3-6 weeks after initiating therapy to enable titration and ensure the patient is not applying an excessive dose. If the dose is increased, based on clinical response and blood level, total testosterone should be repeated within 6 weeks. Different preparations will have different absorption rates and hence different pharmacokinetics that determine when the peak blood level is achieved. As this is unknown for most products in women, the goal of testing blood levels is to prevent excessive dosing but not to treat to a target blood level of testosterone. Clinicians should ensure that the total testosterone does not significantly exceed the upper limit of the reference range for normal premenopausal women indicated by the 
patient's laboratory. When testosterone levels are maintained in the premenopausal range, androgenic side effects are rare. At supraphysiological levels testosterone can cause acne, hirsutism, deepening of the voice, and androgenic alopecia. ${ }^{73}$ Based on available safety data, if the level is supraphysiological, even in the absence of androgenic side effects, the patient should be instructed to titrate down the dose with a repeat blood test after 2-3 weeks. Serum testosterone concentrations should be monitored every 4-6 months once stable levels are achieved to screen for overuse with the inherent downstream androgenic consequences. ${ }^{15,73}$

In women whose total testosterone levels are in the upper end of the physiologic range and not experiencing improvement of HSDD symptoms, calculating free testosterone might provide insight to the lack of response to therapy. An online free and bioavailable testosterone calculator, utilizing total testosterone and SHBG values, is available at http:// www.issam.ch/freetesto.htm. ${ }^{152}$ If elevated SHBG (and resultant decreased free testosterone) is deemed to be related to the lack of efficacy of testosterone therapy, the contributing factors should be addressed; if modification is not possible, the clinician should use a different approach to managing the woman's HSDD. ${ }^{15}$ Increasing the woman's testosterone dose and total testosterone levels beyond physiologic range to "overcome" elevated SHBG is a clinical strategy that is not supported by current evidence on androgen metabolism, AR and intracrine effects, and short- and long-term safety. ${ }^{83,89,90}$ In countries where tibolone is available, it can be used as an alternative MHT in women with high SHBG as tibolone lowers SHBG by approximately $45 \%$.

Patients should be assessed for signs of androgen excess including acne (oily skin), hirsutism (increased facial hair), and androgenic alopecia (thinning of scalp hair). If side effects occur, the dose of testosterone should usually be decreased. Occasionally, androgenic side effects may occur despite standard dosing or normal serum levels of total testosterone or both. This circumstance may occur with low concentrations of SHBG, which may be associated with type 2 diabetes, metabolic syndrome, and/or obesity. ${ }^{153,154}$

Measurement of liver function and a fasting lipid profile is recommended before initiating treatment. Although these tests are unaffected by physiologic doses of transdermal testosterone, liver disease and hyperlipidemia are contraindications to testosterone therapy.

Earlier guidelines recommended annual breast and pelvic examinations, annual mammography, and evaluation of abnormal bleeding, as well as periodic monitoring of lipid profile, liver function tests, and complete blood count. ${ }^{155}$ Given the available safety data, it is reasonable to perform clinical surveillance in accordance with contemporary country-specific guidelines and laboratory testing semiannually, as well as the first year and annually each year thereafter.

\section{Cost and acquisition of testosterone products}

As there are no systemic testosterone products approved by any national regulator except in Australia, prescriptions written for testosterone are usually considered off-label and patients will be required to pay the cost "out of pocket." In fact, using insurance can result in even higher costs. Although many products are used off-label with little scrutiny or prior authorization, testosterone is frequently excluded from such use when prescribed for women. Insurance coverage and reimbursement vary worldwide depending on the health-care system's payment for off-label prescriptions. Given these insurance coverage issues, it may be advantageous for the patient to use a cost-saving strategy such as a downloaded coupon or mobile application for finding discounts. 2 of the most popular in the United States are GoodRx (www.goodrx .com) and SingleCare (www.singlecare.com). Still, "sticker shock" can occur when a patient endeavors to acquire $1 \%$ testosterone gel in individual resealable tubes because the minimum purchase is 30 tubes (a monthly box for a man) and the woman may not realize this amount will typically last 10-12 months. AndroFeme, the 1\% testosterone cream $(10 \mathrm{mg} / \mathrm{mL})$ approved in Australia for use in postmenopausal women, can be acquired over the internet. Licensed practitioners outside Australia need to register at www.doctordirect .com.au to prescribe for direct delivery to patients.

\section{Is DHEA effective for the treatment of HSDD?}

Given that DHEA is an important precursor for extragonadal synthesis of estrogen and testosterone in postmenopausal women, that the production declines with increasing age, and that studies have demonstrated the association of low levels of DHEA with low desire, ${ }^{4,83}$ oral systemic DHEA has been investigated as a possible treatment for HSDD in postmenopausal women. However, evidence is sparse and inconsistent, resulting from heterogeneous study designs, many of which were limited by small sample sizes and the use of nonvalidated instruments to measure sexual function. ${ }^{73}$ Larger studies using validated instruments for the measurement of sexual function have not shown improvement in sexual function in postmenopausal women treated with DHEA. $^{73,156}$ A meta-analysis in 2014 found that DHEA therapy did not improve sexual desire or other aspects of sexual function in postmenopausal women with normal adrenal function. ${ }^{118}$ Therefore, oral DHEA cannot be recommended for the treatment of HSDD in postmenopausal women with normal adrenal function. ${ }^{6}$

In contrast, local vaginal DHEA has only been effective for the treatment of dyspareunia because of vulvovaginal atrophy. An intravaginal DHEA formulation Prasterone (Intrarosa, Endoceutics) has been approved by the FDA and Health Canada for the treatment of dyspareunia due to menopause. ${ }^{157,158}$

\section{Conclusion}

Government-approved testosterone therapy for women is not currently available worldwide, presumably because of the lack of long-term efficacy and safety data and the barriers to product development and approval. Systemic transdermal testosterone therapy is recommended by the Global Position Statement for postmenopausal women; in this Clinical Practice Guideline, testosterone is recommended for postmenopausal and late reproductive age women with HSDD. Current available research supports a moderate therapeutic benefit. Available safety data show no serious adverse events with physiologic testosterone use, but long-term safety has not been established. ${ }^{5}$ Using established clinical guidance, such as the ISSWSH POC for the management of HSDD, ${ }^{15}$ the diagnosis should include a thorough biopsychosocial 
clinical assessment that leads to identification, modification, and management of contributing factors before testosterone therapy is considered. Before initiation of therapy, patients should receive informed consent, and shared decisionmaking should involve a comprehensive discussion of offlabel use, as well as benefits and risks. ${ }^{140}$ A total testosterone level should not be used to diagnose HSDD. Total testosterone is recommended as the best available measure, rather than free or bioavailable testosterone or FAI. Transdermal formulations can be used cautiously with dosing appropriate for women, and signs of androgen excess and total testosterone levels must be monitored routinely to maintain women in the physiologic premenopausal range. The Global Position Statement, the National Academies of Science, Engineering, and Medicine Report, and this Clinical Practice Guideline recommend against the use of compounded testosterone. 6,138

Although the FDA and other government agencies acknowledge the treatment of HSDD in postmenopausal women as a significant unmet need, barriers to regulatory approval for a female testosterone product are formidable. Most important is the lack of guidance as to the types of studies, end points, results, and safety data required for approval, in contrast to the clear pathway for testosterone products for men. ${ }^{159}$ More clinical trial evidence demonstrating longer and more robust testosterone safety data is integral to the development, submission, and regulatory approval of testosterone preparations specifically indicated and designed for women.

\section{Acknowledgments}

These Guidelines have been copublished with permission in The Journal of Sexual Medicine, Journal of Women's Health, and Climacteric. The authors would like to thank Tessa Benitez, Executive Director of ISSWSH.

\section{Statement of Authorship}

Sharon J. Parish: Conceptualization, Investigation, Writing - Original Draft, Writing - Review \& Editing, Supervision, Project Administration; James A. Simon: Conceptualization, Investigation, Writing - Original Draft, Writing - Review \& Editing, Supervision, Project Administration; Susan R. Davis: Investigation, Writing - Original Draft, Writing - Review \& Editing; Annamaria Giraldi: Investigation, Writing - Original Draft, Writing - Review \& Editing; Irwin Goldstein: Investigation, Writing - Original Draft, Writing - Review \& Editing; Sue W. Goldstein: Investigation, Writing - Original Draft, Writing - Review \& Editing, Supervision, Project Administration, Funding Acquisition; Noel N. Kim: Investigation, Writing - Original Draft, Writing - Review \& Editing; Sheryl A. Kingsberg: Investigation, Writing - Original Draft, Writing - Review \& Editing; Abraham Morgentaler: Investigation, Writing - Original Draft, Writing - Review \& Editing; Rossella E. Nappi: Investigation, Writing - Original Draft, Writing - Review \& Editing; Kwangsung Park: Investigation, Writing - Original Draft, Writing - Review \& Editing; Cynthia A. Stuenkel: Investigation, Writing - Original Draft, Writing - Review \& Editing; Abdulmaged M. Traish: Investigation, Writing - Original Draft, Writing - Review \& Editing; Linda Vignozzi: Investigation, Writing - Original Draft, Writing - Review \& Editing.

\section{Author Disclosure Statement}

SJP: Consultant: AMAG Pharmaceuticals, Strategic Science \& Technologies; Writing support, no compensation: AMAG Pharmaceuticals, Sprout Pharmaceuticals, TherapeuticsMD; JAS: AbbVie, Inc., AMAG Pharmaceuticals, Inc., Bayer Healthcare LLC., CEEK Enterprises, LLC., Covance Inc., Daré Bioscience, Duchesnay USA, Endoceutics, Inc., GTx, Inc., Hologic Inc., Ipsen, KaNDy/ NeRRe Therapeutics Ltd, Mitsubishi Tanabe Pharma Development America, Inc., Madorra Pty Ltd, Myovant Sciences, ObsEva SA, Shionogi Inc., Sprout2 Inc., TherapeuticsMD, Viveve Medical; Stockholder (direct purchase) in Sermonix Pharmaceuticals; SRD: Honoraria: Besins Healthcare, Pfizer Australia; Consultant: Mayne Pharmaceuticals, Lawley Pharmaceuticals, Que Oncology; Institutional Grant: Que Oncology research; AG: Consultant: Eli Lilly, Palatin. Speakers Bureau: Pfizer, Astellas; IG: Research: Marius, AbbVie, Consultant: Lipocine, Speakers Bureau: Clarus; SWG: Research: Marius, AbbVie; NNK: None; SAK: AMAG, Astellas, Daré, Duchesney, Emotional Brain, Endoceutics, Ovoca, Lupin, Materna, Palatin Technologies, Pfizer, Sprout, Strategic Science Technologies, TherapeuticsMD, Viveve, Mitsubishi Tanabe NA; AM: Acerus, Antares, Clarus, Research: Endo; REN: Consultant/ speaker: Astellas, Bayer HealthCare AG, Exceltis, Fidia, Merck Sharpe \& Dohme, Novo Nordisk, Palatin Technologies, Pfizer Inc., Shionogi Limited and Theramex; KP: None; CAS: None; AT: None; LV: Research: Lipocine, Bayer, Kyowa Kirin.

\section{Funding Information}

ISSWSH, a portion of which came from an unrestricted educational grant from Lawley Pharmaceuticals. Susan R. Davis is an NHMRC Senior Principal Research Fellow (Grant No. 1135843).

\section{References}

1. Abdallah R, Simon JA. Testosterone therapy in women: its role in the management of hypoactive sexual desire disorder. Int J Impot Res 2007;19:458-463.

2. Hubayter Z, Simon JA. Testosterone therapy for sexual dysfunction in postmenopausal women. Climacteric 2008; 11:181-191.

3. Krapf J, Simon JA. The role of testosterone in the management of hypoactive sexual desire disorder in postmenopausal women. Maturitas 2009;63:213-219.

4. Davis S, Wahlin-Jacobsen S. Testosterone in women-the clinical significance. Lancet Diabetes Endocrinol 2015;3: 980-992.

5. Islam RM, Bell RJ, Green S, et al. Safety and efficacy of testosterone for women: a systematic review and metaanalysis of randomised controlled trial data. Lancet Diabetes Endocrinol 2019;7:754-766.

6. Davis SR, Baber R, Panay N, et al. Global consensus position statement on the use of testosterone therapy for women. J Sex Med 2019;16:1331-1337.

7. Davis SR, Baber R, Panay N, et al. Global consensus position statement on the use of testosterone therapy for women. Climacteric 2019;22:429-434.

8. Davis SR, Baber R, Panay N, et al. Global consensus position statement on the use of testosterone therapy for women. J Clin Endocrinol Metab 2019;104:4660-4666. 
9. Davis SR, Baber R, Panay N, et al. Global consensus position statement on the use of testosterone therapy for women. Maturitas 2019;128:89-93.

10. Simon JA, Kapner MD. The saga of testosterone for menopausal women at the Food and Drug Administration (FDA). J Sex Med 2020;17:826-829.

11. Braunstein G, Sundwall DA, Katz M, et al. Safety and efficacy of a testosterone patch for the treatment of hypoactive sexual desire disorder in surgically menopausal women. Arch Intern Med 2005;165:1582-1589.

12. Simon J, Braunstein G, Nachtigall L, et al. Testosterone patch increases sexual activity and desire in surgically menopausal women with hypoactive sexual desire disorder. J Clin Endocrinol Metab 2005;90:5226-5233.

13. Rossouw J, Anderson GL, Prentice RL, et al. Risks and benefits of estrogen plus progestin in healthy postmenopausal women: principal results from the women's health initiative randomized controlled trial. JAMA 2002;288: 321-333.

14. Anderson GL, Limacher M, Assaf AR, et al. Effects of conjugated equine estrogen in postmenopausal women with hysterectomy: the women's health initiative randomized controlled trial. JAMA 2004;291:1701-1712.

15. Clayton A, Goldstein I, Kim NN, et al. The International Society for the Study of Women's Sexual Health process of care for management of hypoactive sexual desire disorder in women. Mayo Clin Proc 2018;93:467-487.

16. Association AP. Diagnostic and statistical manual of mental disorders IV, text revision (DSM-IV-TR). Washington DC: American Psychiatric Association; 2003.

17. American Psychological Association. Diagnostic and statistical manual of mental disorders. Fifth edition: DSM-5. Arlington, VA: American Psychological Association; 2013.

18. Parish SJ, Goldstein AT, Goldstein SW, et al. Toward a more evidence-based nosology and nomenclature for female sexual dysfunctions-part II. J Sex Med 2016;13: 1888-1906.

19. McCabe M, Sharlip ID, Atalla E, et al. Definitions of sexual dysfunctions in women and men: a consensus statement from the fourth international consultation on sexual medicine. J Sex Med 2015;13:135-143.

20. World Health Organization. The ICD-10 classification of mental and behavioural disorders: clinical descriptions and diagnostic guidelines. World Heath Organization. Available at: https://apps.who.int/iris/handle/10665/ 37958. Accessed January 25, 2020.

21. Reed GM, Drescher J, Krueger RB, et al. Disorders related to sexuality and gender identity in the ICD-11: revising the ICD-10 classification based on current scientific evidence, best clinical practices, and human rights considerations. World Psychiatry 2016;15:205-221.

22. Derogatis LR, Clayton A, Rosen R, et al. Do multiple convergent measures of female sexual dysfunction (FSD) support sexual desire and arousal disorders as distinct diagnoses? J Sex Med 2010;7:S142.

23. Clayton A, DeRogatis IR, Rosen R, et al. Do clinical research data support sexual desire and arousal disorders as distinct diagnoses? J Sex Med 2011;7:S143.

24. Simon JA, Davis SR, Althof SE, et al. Sexual well-being after menopause: an International Menopause Society white paper. Climacteric 2018;21:415-427.

25. Shifren J, Monz BU, Russo PA, et al. Sexual problems and distress in United States women: prevalence and correlates. Obstet Gynecol 2008;112:970-978.
26. Nicolosi A, Glasser DB, Kim SC, et al. Sexual behaviour and dysfunction and help-seeking patterns in adults aged 40-80 years in the urban population of Asian countries. BJU Int 2005;95:609-614.

27. Hayes RD, Dennerstein L, Bennett CM, et al. Relationship between hypoactive sexual desire disorder and aging. Fertil Steril 2007;87:107-112.

28. Worseley R, Bell RJ, Gartoulla P, et al. Prevalence and predictors of low sexual desire, sexually related personal distress, and hypoactive sexual desire dysfunction in a community-based sample of midlife women. J Sex Med 2017;14:675-686.

29. Zeleke B, Bell RJ, Billah B, et al. Hypoactive sexual desire dysfunction in community-dwelling older women menopause. Menopause 2017;24:391-399.

30. Hill K. The demography of menopause. Maturitas 1996; 23:113-127.

31. Maclaran K, Panay N. Current concepts in premature ovarian insufficiency. Womens Health (Lond) 2015;11: 169-182.

32. Kingsberg SA, Larkin LC, Liu JH. Clinical effects of early or surgical menopause. Obstet Gynecol 2020;135:853868.

33. Luborsky JL, Meyer P, Sowers MF, et al. Premature menopause in a multi-ethnic population study of the menopause transition. Hum Reprod 2003;18:199-206.

34. Soman M, Huang LC, Cai WH, et al. Serum androgen profiles in women with premature ovarian insufficiency: a systematic review and meta-analysis. Menopause 2019; 26:78-93.

35. Judd HL, Judd GE, Lucas WE, et al. Endocrine function of the postmenopausal ovary: concentration of androgens and estrogens in ovarian and peripheral vein blood. J Clin Endocrinol Metab 1974;39:1020-1024.

36. Shuster LT, Rhodes DJ, Gostout BS, et al. Premature menopause or early menopause: long-term health consequences. Maturitas 2010;65:161-166.

37. Santoro N. Mechanisms of premature ovarian failure. Ann Endocrinol (Paris) 2003;64:87-92.

38. Mahal AS, Rhoads KF, Elliott CS, et al. Inappropriate oophorectomy at time of benign premenopausal hysterectomy. Menopause 2017;24:947-953.

39. Moore B, Steiner CA, David PH, et al. Trends in hysterectomies and oophorectomies in hospital inpatient and ambulatory settings, 2005-2013. Statistical Brief \#214(November). Rockville, MD: Agency for Healthcare Research and Quality; 2016.

40. Novetsky AP, Boyd LR, Curtin JP. Trends in bilateral oophorectomy at the time of hysterectomy for benign disease. Obstet Gynecol 2011;118:1280-1286.

41. Sarrel PM, Sullivan SD, Nelson LM. Hormone replacement therapy in young women with surgical primary ovarian insufficiency. Fertil Steril 2016;106:1580-1587.

42. Davison S, Bell R, Donath S, et al. Androgen levels in adult females: changes with age, menopause, and oophorectomy. J Clin Endocrinol Metab 2005;90:3847-3853.

43. Arlt W. Androgen therapy in women. Eur J Endocrinol 2006;154:1-11.

44. Hodgson T, Braunstein GD. Physiological effects of androgens in women. In: Azziz R, Nestler JE, Deqailly D, eds. Contemporary endocrinology: androgen excess disorders in women: polycystic ovary syndrome and other disorders, second edition. Totowa: NJ Humana Press Inc; 2007. p. 49-61. 
45. Lobo RA. Androgens in postmenopausal women: production, possible role, and replacement options. Obstet Gynecol Surv 2001;56:361-376.

46. Skiba MA, Bell RJ, Islam RM, et al. Androgens during the reproductive years: what is normal for women? J Clin Endocrinol Metab 2019;104:5382-5392.

47. Davis S, Braunstein GD. Efficacy and safety of testosterone in the management of hypoactive sexual desire disorder in postmenopausal women. J Sex Med 2012;9: 1134-1148.

48. Braunstein GD, Reitz RE, Buch A, et al. Testosterone reference ranges in normally cycling healthy premenopausal women. J Sex Med 2011;8:2924-2934.

49. Bertin J, Dury AY, Ouellet J, et al. Localization of the androgen-synthesizing enzymes, androgen receptor, and sex steroids in the vagina: possible implications for the treatment of postmenopausal sexual dysfunction. J Sex Med 2014;11:1949-1961.

50. Berman JR, Almeida FG, Jolin J, et al. Correlation of androgen receptors, aromatase, and 5-alpha reductase in the human vagina with menopausal status. Fertil Steril 2003;79:925-931.

51. Chang C, Lee SO, Wang RS, et al. Androgen receptor (AR) physiological roles in male and female reproductive systems: lessons learned from AR-knockout mice lacking AR in selective cells. Biol Reprod 2013;89:21.

52. Davey RA, Grossmann M. Androgen receptor structure, function and biology: from bench to bedside. Clin Biochem Rev 2016;37:3-15.

53. Thomas P. Membrane androgen receptors unrelated to nuclear steroid receptors. Endocrinology 2019;160:772-781.

54. Bulldan A, Dietze R, Shihan M, et al. Non-classical testosterone signaling mediated through ZIP9 stimulates claudin expression and tight junction formation in Sertoli cells. Cell Signal 2016;28:1075-1085.

55. Huo YN, Yeh SD, Lee WS. Androgen receptor activation reduces the endothelial cell proliferation through activating the cSrc/AKT/p38/ERK/NF $\kappa$ B-mediated pathway. J Steroid Biochem Mol Biol 2019;194:105459.

56. Höfer P, Lanzenberger R, Kasper S. Testosterone in the brain: neuroimaging findings and the potential role for neuropsychopharmacology. Eur Neuropsychopharmacol 2013;23:79-88.

57. Celec P, Ostatnikova D, Hodosy J. On the effects of testosterone on brain behavioral functions. Front Neurosci 2015;9:12.

58. Traish AM, Botchevar E, Kim NN. Biochemical factors modulating female genital sexual arousal physiology. J Sex Med 2010;7:2925-2946.

59. Sharma UR, Rissman EF. Testosterone implants in specific neural sites activate female sexual behaviour. J Neuroendocrinol 1994;6:423-432.

60. Locklear MN, Michaelos M, Collins WF, et al. Gonadectomy but not biological sex affects burst-firing in dopamine neurons of the ventral tegmental area and in prefrontal cortical neurons projecting to the ventral tegmentum in adult rats. Eur J Neurosci 2017;45:106-120.

61. Wainwright SR, Lieblich SE, Galea LA. Hypogonadism predisposes males to the development of behavioural and neuroplastic depressive phenotypes. Psychoneuroendocrinology 2011;36:1327-1341.

62. Roselli CE, Resko JA. Aromatase activity in the rat brain: hormonal regulation and sex differences. J Steroid Biochem Mol Biol 1993;44:499-508.
63. Maseroli E, Santangelo A, Lara-Fontes B, et al. The nonaromatizable androgen dihydrotestosterone (DHT) facilitates sexual behavior in ovariectomized female rats primed with estradiol. Psychoneuroendocrinology 2020; 115:104606.

64. Dennerstein L, Randolph J, Taffe J, et al. Hormones, mood, sexuality, and the menopausal transition. Fertil Steril 2002;77 Suppl 4:S42-S48.

65. Roney JR, Simmons ZL. Hormonal predictors of sexual motivation in natural menstrual cycles. Horm Behav 2013; 63:636-645.

66. Bramen JE, Hranilovich JA, Dahl RE, et al. Sex matters during adolescence: testosterone-related cortical thickness maturation differs between boys and girls. PLoS One 2012;7:e33850.

67. Cappelletti M, Wallen K. Increasing women's sexual desire: the comparative effectiveness of estrogens and androgens. Horm Behav 2016;78:178-193.

68. Nastri C, Lara LA, Ferriani RA, et al. Hormone therapy for sexual function in perimenopausal and postmenopausal women. Cochrane Database Syst Rev 2013;5: CD009672.

69. Davis SR, Goldstat R, Papalia MA, et al. Effects of aromatase inhibition on sexual function and well-being in postmenopausal women treated with testosterone: a randomized, placebo-controlled trial. Menopause 2006;13:37-45.

70. Levin RJ, Both S, Georgiadis J, et al. The physiology of female sexual function and the pathophysiology of female sexual dysfunction (Committee 13A). J Sex Med 2016;13: 733-759.

71. Park K, Kim N. Anatomy and physiology of arousal. In: Goldstein I, Clayton AH, Goldstein AT, et al., editors. Textbook of female sexual function and dysfunction: diagnosis and treatment. Oxford: Wiley Blackwell; 2018. p. 107-125.

72. Levin R. The pharmacology of the human female orgasm-its biological and physiological backgrounds. Pharmacol Biochem Behav 2014;121:62-70.

73. Davis S, Worsley R, Miller KK, et al. Androgens and female sexual function and dysfunction-findings from the fourth international consultation of sexual medicine. J Sex Med 2016;13:168-178.

74. Traish A, Vignozzi L, Goldstein I, et al. Role of androgens and estrogens in female genitourinary tract structure and function: implications in the genitourinary syndrome of menopause. Sex Med Rev 2018;5:558-571.

75. Simon JA, Goldstein I, Kim NN, et al. The role of androgens in the treatment of genitourinary syndrome of menopause (GSM): International Society for the Study of Women's Sexual Health (ISSWSH) expert consensus panel review. Menopause 2018;25:837-847.

76. Vignozzi L, Filippi S, Morelli A, et al. Testosterone/estradiol ratio regulates NO-induced bladder relaxation and responsiveness to PDE5 inhibitors. J Sex Med 2012;9: 3028-3040.

77. Labrie F, Martel C, Pelletier G. Is vulvovaginal atrophy due to a lack of both estrogens and androgens? Menopause 2017;24:452-461.

78. Comeglio P, Cellai I, Filippi S, et al. Differential effects of testosterone and estradiol on clitoral function: an experimental study in rats. J Sex Med 2016;13:1858-1871.

79. Traish AM, Kim N, Min K, et al. Role of androgens in female genital sexual arousal: receptor expression, structure, and function. Fertil Steril 2002; 77 Suppl 4:S11-S18. 
80. Bachmann G, Bancroft J, Braunstein G, et al. Female androgen insufficiency: the Princeton consensus statement on definition, classification, and assessment. Fertil Steril 2002;77:660-665.

81. Burger HG, Dudley EC, Cui J, et al. A prospective longitudinal study of serum testosterone, dehydroepiandrosterone sulfate, and sex hormone-binding globulin levels through the menopause transition. J Clin Endocrinol Metab 2000;85:2832-2838.

82. Davis SR, Bell RJ, Robinson PJ, et al. Testosterone and estrone increase from the age of 70 years: findings from the sex hormones in older women study. J Clin Endocrinol Metab 2019;104:6291-6300.

83. Wåhlin-Jacobsen S, Pedersen AT, Kristensen E, et al. Is there a correlation between androgens and sexual desire in women? J Sex Med 2015;12:358-373.

84. Zheng J, Islam R, Skiba MA, et al. Associations between androgens and sexual function in premenopausal women; a cross-sectional study. Lancet Diabetes Endocrinol 2020; 8:693-702.

85. Randolph Jr. JF, Zheng H, Avis NE, et al. Masturbation frequency and sexual function domains are associated with serum reproductive hormone levels across the menopausal transition. J Clin Endocrinol Metab 2015;100:258-266.

86. Wåhlin-Jacobsen S, Kristensen E, Pedersen AT, et al. Androgens and psychosocial factors related to sexual dysfunctions in premenopausal women(*): (*)2016 ISSM female sexual dysfunction prize. J Sex Med 2017;14:366-379.

87. Leiblum S, Koochaki PE, Rodenberg CA, et al. Hypoactive sexual desire disorder in postmenopausal women: US results from the women's international study of health and sexuality (WISHeS). Menopause 2006;13:46-56.

88. Davis S, Davison SL, Donath S, et al. Circulating androgen levels and self-reported sexual function in women. JAMA 2005;294:91-96.

89. Labrie F. Extragonadal synthesis of sex steroids: intracrinology. Ann Endocrinol (Paris) 2003;64:95-107.

90. Wåhlin-Jacobsen S, Flanagan JN, Pedersen AT, et al. Androgen receptor polymorphism and female sexual function and desire. J Sex Med 2018;15:1537-1546.

91. Krapf JM, Simon JA. A sex-specific dose-response curve for testosterone: could excessive testosterone limit sexual interaction in women? Menopause 2017;24:462-470.

92. Guay A, Jacobson J, Munarriz R, et al. Serum androgen levels in healthy premenopausal women with and without sexual dysfunction: part B: reduced serum androgen levels in healthy premenopausal women with complaints of sexual dysfunction. Int J Impot Res 2004;16:121-129.

93. Wilke TJ, Utley DJ. Total testosterone, free-androgen index, calculated free testosterone, and free testosterone by analog RIA compared in hirsute women and in otherwise-normal women with altered binding of sexhormone-binding globulin. Clin Chem 1987;33:13721375.

94. Rosner W, Auchus RJ, Azziz R, et al. Position statement: utility, limitations, and pitfalls in measuring testosterone: an Endocrine Society position statement. J Clin Endocrinol Metab 2007;92:405-413.

95. Rosner W, Vesper H. Toward excellence in testosterone testing: a consensus statement. J Clin Endocrinol Metab 2010;95:4542-4548.

96. Goldman AL, Bhasin S, Wu FCW, et al. A reappraisal of testosterone's binding in circulation: physiological and clinical implications. Endocr Rev 2017;38:302-324.
97. Somboonporn W, Davis S, Seif M, et al. Testosterone for peri- and postmenopausal women. Cochrane Database Syst Rev 2005:CD004509.

98. Achilli C, Pundir J, Ramanathan P, et al. Efficacy and safety of transdermal testosterone in postmenopausal women with hypoactive sexual desire disorder: a systematic review and meta-analysis. Fertil Steril 2017;107: 475-482.e415.

99. Burger HG, Hailes J, Nelson J, et al. Effect of combined implants of estradiol and testosterone on libido in postmenopausal women. BMJ 1987;294:936-937.

100. Davis SR, McCloud PI, Strauss BJG, et al. Testosterone enhances estradiol's effects on postmenopausal bone density and sexuality. Maturitas 1995;21:227-236.

101. Lobo R, Rosen RC, Yang H-M, et al. Comparative effects of oral esterified estrogens with and without methyl testosterone on endocrine profiles and dimensions of sexual function in postmenopausal women with hypoactive sexual desire. Fertil Steril 2003;79:1341-1352.

102. Floter A, Nathorst-Boos J, Carlstrom K, et al. Addition of testosterone to estrogen replacement therapy in oophorectomized women: effects on sexuality and well-being. Climacteric 2002;5:357.

103. Buckler HM, Robertson WR, Wu FC. Which androgen replacement therapy for women? J Clin Endocrinol Metab 1998;83:3920-3924.

104. Davis SR, Moreau M, Kroll R, et al. Testosterone for low libido in menopausal women not taking estrogen therapy. N Engl J Med 2008;359:2005-2017.

105. Fooladi E, Reuter SE, Bell RJ, et al. Pharmacokinetics of a transdermal testosterone cream in healthy postmenopausal women. Menopause 2015;22:44-49.

106. Kingsberg S, Shifren J, Wekselman K, et al. Evaluation of the clinical relevance of benefits associated with transdermal testosterone treatment in postmenopausal women with hypoactive sexual desire disorder. J Sex Med 2007; 4(4 Pt 1):1001-1008.

107. Goldstat R, Briganti E, Tran J, et al. Transdermal testosterone improves mood, well being and sexual function in premenopausal women. Menopause 2003;10:390 398.

108. Fooladi E, Bell RJ, Jane F, et al. Testosterone improves antidepressant-emergent loss of libido in women: findings from a randomized, double-blind, placebo-controlled trial. J Sex Med 2014;11:831-839.

109. Davis SR, Papalia MA, Norman RJ, et al. Safety and efficacy of a testosterone metered-dose transdermal spray for treatment of decreased sexual satisfaction in premenopausal women: a placebo-controlled randomized, dose-ranging study. Ann Intern Med 2008;148:569577.

110. Barrett-Connor E, Young R, Notelovitz M, et al. A twoyear, double-blind comparison of estrogen-androgen and conjugated estrogens in surgically menopausal women. Effects on bone mineral density, symptoms and lipid profiles. J Reprod Med 1999;44:1012-1020.

111. Elraiyah T, Sonbol MB, Wang Z, et al. Clinical review: the benefits and harms of systemic testosterone therapy in postmenopausal women with normal adrenal function: a systematic review and meta-analysis. J Clin Endocrinol Metab 2014;99:3543-3550.

112. Boyd PR, Mark GJ. Multiple hepatic adenomas and a hepatocellular carcinoma in a man on oral methyl testosterone for eleven years. Cancer 1977;40:1765-1770. 
113. Pharmaceuticals V. Android C-III package insert. Available at: https://www.accessdata.fda.gov/drugsatfda_docs/ label/2015/083976s032lbl.pdf. Accessed May 8, 2020.

114. Nachtigall L, Casson P, Lucas J, et al. Safety and tolerabililty of testosterone patch therapy for up to 4 years in surgically menopausal women receiving oral or transdermal oestrogen. Gynecol Endocrinol 2011;27:39-48.

115. White WB, Grady D, Giudice LC, et al. A cardiovascular safety study of LibiGel (testosterone gel) in postmenopausal women with elevated cardiovascular risk and hypoactive sexual desire disorder. Am Heart J 2012;163:27-32.

116. Pharmaceuticals B. BioSante Pharmaceuticals announces positive LibiGel phase III safety data review and decision to conclude the safety study. Available at: https://www .businesswire.com/news/home/20120904005404/en/Bio Sante-Pharmaceuticals-Announces-Positive-LibiGel\%C2\% AE-Phase-III Accessed June 21, 2019.

117. Wierman M, Arlt W, Basson R, et al. Androgen therapy in women: a reappraisal: an Endocrine Society clinical practice guideline. J Clin Endocrinol Metab 2014;99: 2489-3510.

118. Elraiyah T, Sonbol MB, Wang Z, et al. Clinical review: the benefits and harms of systemic dehydroepiandrosterone (DHEA) in postmenopausal women with normal adrenal function: a systematic review and meta-analysis. J Clin Endocrinol Metab 2014;99:3536-3542.

119. Jayasena CN, Alkaabi FM, Liebers CS, et al. A systematic review of randomized controlled trials investigating the efficacy and safety of testosterone therapy for female sexual dysfunction in postmenopausal women. Clin Endocrinol (Oxf) 2019;90:391-414.

120. Glaser RL, Dimitrakakis C. Reduced breast cancer incidence in women treated with subcutaneous testosterone, or testosterone with anastrozole: a prospective, observational study. Maturitas 2013;76:342-349.

121. Clayton A, Goldfischer ER, Goldstein I, et al. Validation of the decreased sexual desire screener (DSDS): a brief diagnostic instrument for generalized acquired female hypoactive sexual desire disorder (HSDD). J Sex Med 2009;6:730-738.

122. Kingsberg SA, Althof S, Simon JA, et al. Female sexual dysfunction-medical and psychological treatments, Committee 14. J Sex Med 2017;14:1463-1491.

123. Advisory Committee Briefing Document Intrinsa ${ }^{\circledR}$ (testosterone transdermal system) 2 December 2004. NDA No. 21-769 Procter \& Gamble Pharmaceuticals, Inc., Advisory Committee for Reproductive Health Drugs. Food and Drug Administration, USA. Available at: https:// wayback.archive-it.org/7993/20170405114619/https://www .fda.gov/ohrms/dockets/ac/04/briefing/2004-4082B1_01_ A-P\&G-Intrinsa.pdf Accessed April 20, 2019.

124. Baber RJ, Panay N, Fenton A. 2016 IMS recommendations on women's midlife health and menopause hormone therapy. Climacteric 2016;19:109-150.

125. The 2017 hormone therapy position statement of The North American Menopause Society. Menopause 2017; 24:728-753.

126. Hamoda H. The British Menopause Society and women's health concern recommendations on the management of women with premature ovarian insufficiency. Post Reprod Health 2017;23:22-35.

127. Committee opinion no. 698: hormone therapy in primary ovarian insufficiency. Obstet Gynecol 2017;129:e134e141.
128. Webber L, Davies M, Anderson R, et al. ESHRE guideline: management of women with premature ovarian insufficiency. Hum Reprod 2016;31:926-937.

129. Vujovic S, Brincat $M$, Erel $T$, et al. EMAS position statement: managing women with premature ovarian failure. Maturitas 2010;67:91-93.

130. Nappi RE, Cucinella L, Martini E, et al. Sexuality in premature ovarian insufficiency. Climacteric 2019;22: 289-295.

131. Shifren JL, Desindes S, McIlwain M, et al. A randomized, open-label, crossover study comparing the effects of oral versus transdermal estrogen therapy on serum androgens, thyroid hormones, and adrenal hormones in naturally menopausal women. Menopause 2007;14:985-994.

132. Taylor HS, Tal A, Pal L, et al. Effects of oral vs transdermal estrogen therapy on sexual function in early postmenopause: ancillary study of the kronos early estrogen prevention study (KEEPS). JAMA Intern Med 2017;177:1471-1479.

133. Davis SR, Bitzer J, Giraldi A, et al. Change to either a nonandrogenic or androgenic progestin-containing oral contraceptive preparation is associated with improved sexual function in women with oral contraceptive-associated sexual dysfunction. J Sex Med 2013;10:3069-3079.

134. Both S, Lew-Starowicz M, Luria M, et al. Hormonal contraception and female sexuality: position statements from the European Society of Sexual Medicine (ESSM). J Sex Med 2019;16:1681-1695.

135. Braunstein GD. Safety of testosterone treatment in postmenopausal women. Fertil Steril 2007;88:1-17.

136. Shifren JL, Braunstein GD, Simon JA, et al. Transdermal testosterone treatment in women with impaired sexual function after oophorectomy. N Engl J Med 2000;343: 682-688.

137. Grober ED, Garbens A, Božović A, et al. Accuracy of testosterone concentrations in compounded testosterone products. J Sex Med 2015;12:1381-1388.

138. National Academies of Sciences Engineering and Medicine. The clinical utility of compounded bioidentical hormone therapy: a review of safety, effectiveness, and use. The National Academies Press; 2020. Available at: https://www.nap.edu/catalog/25791/the-clinical-utility-ofcompounded-bioidentical-hormone-therapy-a-review Accessed September 23, 2020.

139. Nathorst-Boos J, Floter A, Jarkander-Rolff M, et al. Treatment with percutanous testosterone gel in postmenopausal women with decreased libido-effects on sexuality and psychological general well-being. Maturitas 2006;53: 11-18.

140. Vegunta S, Kling JM, Kapoor E. Androgen therapy in women. J Womens Health (Larchmt) 2020;29:57-64.

141. Parish SJ, Nappi RE, Kingsberg S. Perspectives on counseling patients about menopausal hormone therapy: strategies in a complex data environment. Menopause 2018;25:937-949.

142. Guay A, Munarriz R, Jacobson J, et al. Serum androgen levels in healthy premenopausal women with and without sexual dysfunction: part A. Serum androgen levels in women aged 20-49 years with no complaints of sexual dysfunction. Int J Impot Res 2004;16:112-120.

143. Platz EA, Barber JR, Chadid S, et al. Nationally representative estimates of serum testosterone concentration in never-smoking, lean men without aging-associated comorbidities. J Endocr Soc 2019;3:1759-1770. 
144. Lewis T, Goldstein I. Transfer of topical testosterone preparations to children or spouses. J Sex Med 2009;6: 2649-2652.

145. Rosen R, Brown C, Heiman J, et al. The female sexual function index (FSFI): a multidimensional self-report instrument for the assessment of female sexual function. J Sex Marital Ther 2000;26:191-208.

146. Derogatis L, Clayton A, Lewis-D'Agostino D, et al. Validation of the female sexual distress scale-revised for assessing distress in women with hypoactive sexual desire disorder. J Sex Med 2008;5:357-364.

147. Kennedy RG, Davies T, Al-Azzawi F. Sexual interest in postmenopausal women is related to 5alpha-reductase activity. Hum Reprod 1997;12:209-213.

148. Shifren J, Davis S, Moreau M, et al. Testosterone patch for the treatment of hypoactive sexual desire disorder in naturally menopausal women: results from the INTIMATE NM1 study. Menopause 2006;13:770-779.

149. Schmidt U, Nygaard B, Jensen EW, et al. Peripheral markers of thyroid function: the effect of T4 monotherapy vs T4/T3 combination therapy in hypothyroid subjects in a randomized crossover study. Endocr Connect 2013;2:55-60.

150. Selva D, Hammond GL. Thyroid hormones act indirectly to increase sex hormone-binding globulin production by liver via hepatocyte nuclear factor-4alpha. J Mol Endocrinol 2009;43:19-27.

151. Hampl R, Kancheva R, Hill M, et al. Interpretation of sex hormone-binding globulin levels in thyroid disorders. Thyroid 2003;13:755-760.

152. Hormonology department UHoG, Belgium. Free \& bioavailable testosterone calculator. Available at: http://www .issam.ch/freetesto.htm Accessed March 29, 2020.

153. Muka T, Nano J, Jaspers L, et al. Associations of steroid sex hormones and sex hormone-binding globulin with the risk of type 2 diabetes in women: a populationbased cohort study and meta-analysis. Diabetes 2017;66: 577-586.

154. Kische H, Gross S, Wallaschofski H, et al. Clinical correlates of sex hormones in women: the study of health in Pomerania. Metab Clin Exp 2016;65:1286-1296.

155. Basson R, Wierman ME, van Lankveld J, et al. Summary of the recommendations on sexual dysfunctions in women. J Sex Med 2010;7(1 Pt 2):314-326.

156. Peixoto C, Carrilho CG, Barros JA, et al. The effects of dehydroepiandrosterone on sexual function: a systematic review. Climacteric 2017;20:129-137.

157. Portman DJ, Goldstein SR, Kagan R. Treatment of moderate to severe dyspareunia with intravaginal prasterone therapy: a review. Climacteric 2019;22:65-72.

158. Sauer U, Talaulikar V, Davies MC. Efficacy of intravaginal dehydroepiandrosterone (DHEA) for symptomatic women in the peri- or postmenopausal phase. Maturitas 2018;116:79-82.

159. Simon JA. Implementing a successful clinical development program for female sexual dysfunctions (aka how to navigate a regulatory minefield). Maturitas 2011;69: 97-98.

Address correspondence to: Sharon J. Parish, MD New York Presbyterian Hospital/Westchester Behavioral Health Center 21 Bloomingdale Road White Plains, NY 10605

USA

E-mail: shp9079@med.cornell.edu 